Article

\title{
Novel Halogenated Pyrazine-Based Chalcones as Potential Antimicrobial Drugs ${ }^{\dagger}$
}

\author{
Marta Kucerova-Chlupacova ${ }^{1, *}$, Veronika Vyskovska-Tyllova ${ }^{1}$, Lenka Richterova-Finkova ${ }^{1}$, \\ Jiri Kunes ${ }^{2}$, Vladimir Buchta ${ }^{3,4}$, Marcela Vejsova ${ }^{3,4}$, Pavla Paterova ${ }^{3,4}$, Lucia Semelkova ${ }^{1}$, \\ Ondrej Jandourek ${ }^{1}$ and Veronika Opletalova ${ }^{1}$
}

1 Department of Pharmaceutical Chemistry and Pharmaceutical Analysis,

Faculty of Pharmacy in Hradec Kralove, Charles University in Prague, Heyrovskeho 1203,

50005 Hradec Kralove, Czech Republic; good-message@seznam.cz (V.V.-T.);

lenka.finkova@seznam.cz (L.R.-F.); semelkol@faf.cuni.cz (L.S.); jando6aa@faf.cuni.cz (O.J.);

opletalova@faf.cuni.cz (V.O.)

2 Department of Inorganic and Organic Chemistry, Faculty of Pharmacy in Hradec Kralove, Charles University in Prague, Heyrovskeho 1203, 50005 Hradec Kralove, Czech Republic;

kunes@faf.cuni.cz

3 Department of Biological and Medical Sciences, Faculty of Pharmacy in Hradec Kralove, Charles University in Prague, Heyrovskeho 1203, 50005 Hradec Kralove, Czech Republic; vejsova@faf.cuni.cz

4 Department of Clinical Microbiology, University Hospital Hradec Kralove, Sokolska 581, 50005 Hradec Kralove, Czech Republic; vladimir.buchta@fnhk.cz (V.B.); pavla.paterova@fnhk.cz (P.P.)

* Correspondence: kucerom@faf.cuni.cz; Tel.: +420-495-067-373

+ Preliminary Results were Presented at the 8th Central European Conference "Chemistry towards Biology" (CTB-2016), Brno, Czech Republic, 28 August-1 September 2016 (Paper P-38).

Academic Editor: Josef Jampilek

Received: 23 September 2016; Accepted: 14 October 2016; Published: 27 October 2016

Abstract: Chalcones, i.e., compounds with the chemical pattern of 1,3-diphenylprop-2-en-1-ones, exert a wide range of bio-activities, e.g., antioxidant, anti-inflammatory, anticancer, anti-infective etc. Our research group has been focused on pyrazine analogues of chalcones; several series have been synthesized and tested in vitro on antifungal and antimycobacterial activity. The highest potency was exhibited by derivatives with electron withdrawing groups (EWG) in positions 2 and 4 of the ring $B$. As halogens also have electron withdrawing properties, novel halogenated derivatives were prepared by Claisen-Schmidt condensation. All compounds were submitted for evaluation of their antifungal and antibacterial activity, including their antimycobacterial effect. In the antifungal assay against eight strains of selected fungi, growth inhibition of Candida glabrata and Trichophyton interdigitale (formerly T. mentagrophytes) was shown by non-alkylated derivatives with 2-bromo or 2-chloro substitution. In the panel of selected bacteria, 2-chloro derivatives showed the highest inhibitory effect on Staphylococcus sp. In addition, all products were also screened for their antimycobacterial activity against Mycobacterium tuberculosis H37RV My 331/88, M. kansasii My 235/80, M. avium 152/80 and M. smegmatis CCM 4622. Some of the examined compounds, inhibited growth of $M$. kansasii and M. smegmatis with minimum inhibitory concentrations (MICs) comparable with those of isoniazid.

Keywords: pyrazine; chalcone; halogenated; antifungal; antibacterial; antimycobacterial

\section{Introduction}

Chalcones are compounds with the basic scaffold of 1,3-diphenylprop-2-en-1-one, containing ring A and ring B connected by an $\alpha, \beta$-unsaturated keto linker (indicated in red in Figure 1, structure 1). Naturally-occurring chalcones (in the plant kingdom) usually bear hydroxy-, methoxy- or prenyl 
substitutions and might serve as precursors of other flavonoid groups. Chalcones exert a wide range of bio-activities (e.g., antioxidant, anti-inflammatory, anticancer, anti-infective etc.), which were several times reviewed [1-3]. In synthetic chalcones, substitutents can be more varied and benzene rings have been many times replaced with other aryls or heteroaryls [2,3].<smiles>COc1cc(O)c(C(=O)/C=C/c2ccc(Cl)cc2)c(OC)c1</smiles>

1<smiles>COc1cccc(/C=C/C(=O)c2ccc(Br)cc2)c1</smiles>

2<smiles></smiles><smiles>[R]c1ccc(/C=C/C(=O)c2ccc([R])cc2)cc1</smiles>

4a $\mathrm{R}^{1}=\mathrm{SCH}_{3}, \mathrm{R}^{2}=\mathrm{Cl}$

4b $\mathrm{R}^{1}=\mathrm{SCH}_{3}, \mathrm{R}^{2}=\mathrm{Br}$ 4c $\mathrm{R}^{1}=\mathrm{Cl}, \mathrm{R}^{2}=\mathrm{NO}_{2}$<smiles>Cc1c(Cl)ccc2cc(/C=C/C(=O)c3ccc(Br)cc3)c(Cl)nc12</smiles>

Figure 1. Examples of antifungal and antibacterial chalcone derivatives.

Based on our previous results concerning chalcones and the positive effect of electron-withdrawing group (EWG) substitution on their antimicrobial effects [4,5], we decided to prepare pyrazine analogues of chalcones halogenated in the ring B. Substitution of chalcones with halogens emerges also in studies of other authors, e.g., compound 1 inhibited growth of Trichophyton rubrum at minimum inhibitory concentrations (MIC) $12.5 \mu \mathrm{g} / \mathrm{mL}$ [6]. Compound 2 exerted better antifungal activity against dermatophytes (MIC $0.5-25 \mu \mathrm{g} / \mathrm{mL}$ ) than amphotericin $\mathrm{B}$ and ketoconazole [7]. In a study comparing antifungal activity of mono- and dihalogenated 1-(2-hydroxyphenyl)-3-phenylprop-2-en-1-ones (3a) and their corresponding flavonols (3b) [8], it was found that chalcones are more active against Trichophyton longifusus, Aspergillus flavus and Microsporum canis than the flavonols. The most active compound was fluorinated in position 4 of the ring B. Chalcones $4 \mathbf{a}-4 \mathbf{c}$ with halogen substitution in position 4 either in the ring $\mathrm{A}$ or in the ring $\mathrm{B}$ inhibited growth of Candida tropicalis and Aspergillus flavus at a concentration of $2 \mathrm{mg} / \mathrm{mL}$ at least by $98 \%[9]$.

Antibacterial effects of chalcones have also been reviewed [10-13] and importance of searching for new antimicrobial agents due to increasing antibiotic resistance of bacteria and fungi has been highlighted [13]. The substitution of aromatic rings with EWG was emphasized in a quinoline-based series of chalcones in association with antibacterial activity. The most active compound, inhibiting growth of Bacillus subtilis, Staphylococcus aureus, Streptococcus pyogenes, Escherichia coli, Klebsiella aerogenes and Salmonella typhimurium, better than chloramphenicol or ciprofloxacin, is depicted in structure 5 . Supposed mechanism of action was explored in a bacterial gyrase assay [14]. Two potentially antibacterial entities were combined in several series containing halogenated chalcones and variously substituted 2-thioxothiazolidin-4-ones (rhodanines) [15,16] or thiazolidin-2,4-dione [17]. Compounds, that inhibited Staphylococcus sp. comparably to norfloxacin, are drawn as structure 6 [15] and 7 [16] in Figure 2. In the same research group, analogical chalcones with substituted thiazolidinediones $8 \mathbf{a}$ 
and $\mathbf{8 b}$ were synthesized (Figure 2). They inhibited growth of two strains of $S$. aureus similarly to oxacillin and norfloxacin [17].

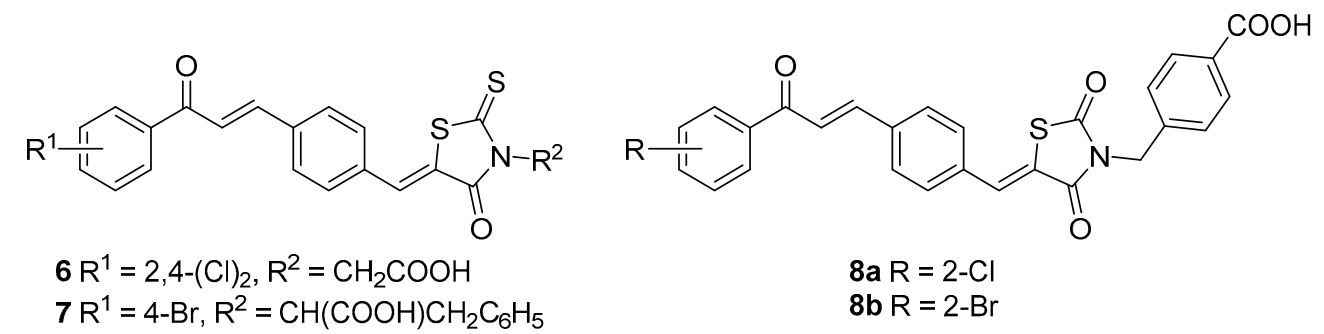<smiles>CC(C)=CCOc1ccc(/C=C/C(=O)c2ccc(OCC=C(C)C)cc2OCC(=O)O)cc1</smiles>

Figure 2. Examples of antibacterial chalcone derivatives.

Halogen substitution has been found to be favorable in antimycobacterial diphenylpropenones [18] and 3-phenyl-1-pyridylpropenones [19]. Chalcones including halogenated derivatives have been described as inhibitors of Mycobacterium tuberculosis phosphatases [20] or inhibitors of fatty acid synthase II [21].

Halogenated chalcones are further mentioned in literature as potential antimalarial [22], anti-inflammatory [23-25], anti-cancer [26] agents, and as potential modulators of multidrug resistance [27].

Sofalcone, explored and marketed in Japan, is a chalcone derivative. Mechanism of its action in ulcer disease is complex, but it possesses bactericidal effect against Helicobacter pylori as well [28].

\section{Results and Discussion}

\subsection{Synthesis}

Preparation of the title compounds is shown in Scheme 1. Pyrazine-2-carbonitrile (10) was used as the starting material. Syntheses of intermediates 11a-11g and 12a-12g were accomplished by formerly described methods [29,30]. Acetylpyrazine 12a and its 5-alkylated congeners $\mathbf{1 2} \mathbf{b}-\mathbf{1 2} \mathbf{g}$ served as starting materials for Claisen-Schmidt condensation with 2- or 4-halogenated aromatic aldehydes affording (2E)-1-(5-unsubstituted or 5-alkyl-pyrazin-2-yl)-3-(halogenphenyl)prop-2-en-1-ones 13a-18g (substitutions indicated in Table 1), according to a previously reported method [31]. Column chromatography, in several cases repeated, was necessary for the separation of the product from the reaction mixture. To obtain analytically pure crystals, recrystallizations from absolute ethanol were needed, which finally resulted in quite low yields.

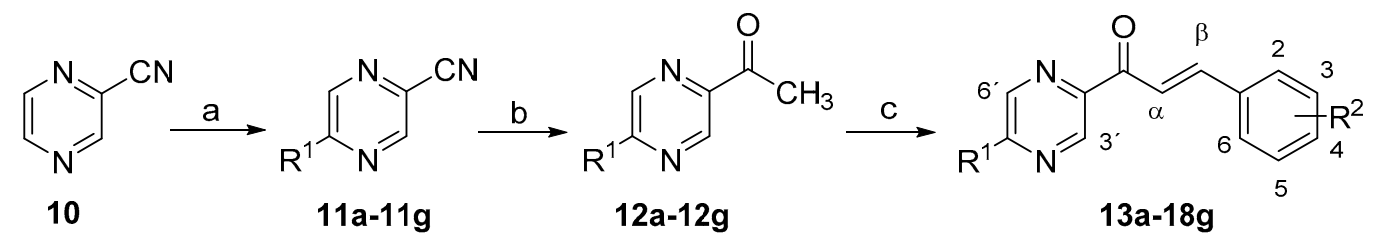

$\mathrm{R}^{1}$ for $\mathbf{1 1}$ and $\mathbf{1 2}=\mathrm{H}$, tert-butyl, isobutyl, butyl, propyl, isopropyl or pentyl; substitutions of structures 13-18 indicated in Table 1

Scheme 1. Synthesis of halogenated pyrazine-based chalcones. Reagents and conditions: (a) aliphatic acid, $\mathrm{AgNO}_{3},\left(\mathrm{NH}_{4}\right)_{2} \mathrm{~S}_{2} \mathrm{O}_{8}$, water, $80{ }^{\circ} \mathrm{C}$; (b) $\mathrm{CH}_{3} \mathrm{MgI}, \mathrm{Et}_{2} \mathrm{O}$; (c) halogenated benzaldehyde, pyridine, $\mathrm{Et}_{2} \mathrm{NH}$. 
Due to difficult purification, only 5 fluorinated derivatives (13a, 13b, 13e, 14a and 14b) were obtained in sufficient amounts. In 2-chloro substituted series, two compounds $15 a$ and $\mathbf{1 5 b}$ were successfully purified and available for biological evaluation. Compounds with chlorine in position 4 of the ring B (16a-16f) were synthesized earlier [5,31]. Influence of compounds 16a-16e on M. tuberculosis H37RV (ATCC 27294) and photosynthetic processes has already been published [32]. However, the accomplishment of their antifungal and antibacterial tests failed due to poor solubility in testing media. For the purpose of the present study, these compounds were newly adjusted by rubbing to be able to pass biological assays. Compound $\mathbf{1 6} \mathbf{f}$ was reported in our previous paper [5]. It displayed neither antifungal nor antimycobacterial activity against M. tuberculosis H37RV (ATCC 27294) [5]. In the present study, we report the results of its antibacterial testing and its effects on the growth of other mycobacteria. All 2-brominated compounds $\mathbf{1 7} \mathbf{a}-\mathbf{1 7 f}$ were obtained without difficulties in acceptable yields $(20 \%-50 \%)$. In contrast, 4 -brominated derivatives $\mathbf{1 8 a}-\mathbf{1 8 g}$ were mostly obtained in small amounts (yields: $2 \%-6 \%)$. (2E)-3-(4-bromophenyl)-1-(5-pentylpyrazin-2-yl)prop-2-en-1-one 18g is the only representative with a longer alkyl in the pyrazine ring since the longer alkyl the more difficult separation of the product from the reaction mixture. This has been observed for both 2-alkylated pyrazine-2-carbonitriles 12 [29,30] and halogenated (2E)-3-phenyl-1-(pyrazin-2-yl)prop-2-en-1-ones reported here.

Table 1. Halogenated pyrazine-based chalcones 13a-18g tested within this work.

\begin{tabular}{|c|c|c|c|c|c|c|c|}
\hline \multirow{2}{*}{$\mathbf{R}^{2}$} & \multicolumn{7}{|c|}{$\mathbf{R}^{1}$} \\
\hline & $\mathbf{H}$ & tert-butyl & isobutyl & butyl & propyl & isopropyl & pentyl \\
\hline $2-\mathrm{F}$ & $13 a$ & $13 b$ & - & - & $13 e$ & - & - \\
\hline $4-F$ & $14 a$ & $14 b$ & - & - & - & - & - \\
\hline $2-\mathrm{Cl}$ & $15 a$ & $15 b$ & - & - & - & - & - \\
\hline $4-\mathrm{Cl}$ & $\begin{array}{c}16 a \\
a\end{array}$ & $16 b^{a}$ & $16 c^{a}$ & $16 d^{a}$ & $16 e^{a}$ & $16 f^{b}$ & - \\
\hline $2-\mathrm{Br}$ & $17 a$ & $17 \mathrm{~b}$ & $17 \mathrm{c}$ & $17 d$ & $17 \mathrm{e}$ & $17 f$ & - \\
\hline $4-\mathrm{Br}$ & $18 a$ & $18 b$ & $18 \mathrm{c}$ & $18 d$ & $18 \mathrm{e}$ & $18 f$ & $18 \mathrm{~g}$ \\
\hline
\end{tabular}

aynthesis, antimycobacterial activity against M. tuberculosis H37RV (ATCC 27294) and influence on photosynthetic processes published previously [32] ${ }^{\mathrm{b}}$ synthesis, antimycobacterial activity against $M$. tuberculosis H37RV (ATCC 27294) and antifungal activity published previously [5].

Identity of compounds was confirmed by melting points, NMR and IR spectra. Proton NMR spectra clearly showed $\left(J_{\mathrm{H \alpha H \beta}}=\mathrm{xx}-\mathrm{yy} \mathrm{Hz}\right)$ that $E$-isomers have been obtained in all cases. Purity of the compounds was verified by TLC and elemental analysis.

\subsection{Evaluation of In Vitro Antifungal Activity}

All prepared compounds, including 16a-16e, were subjected to antifungal assay, only the most active ones are displayed in Table 2. In the panel of selected fungi, inhibition of growth of Trichophyton interdigitale 445 by the non-alkylated compounds was the most notable. That is in accordance with the previous results of our research group in the field of antifungal activity of pyrazine analogues of chalcones [4,5,31]. The derivative with 2-chloro substitution in the phenyl, i.e., ring B (15a) proved the best activity (MIC $3.9 \mu \mathrm{mol} / \mathrm{L}$ after $24 \mathrm{~h}$ incubation), comparable with that of systematically used antimycotic fluconazole (MIC $6.51 \mu \mathrm{mol} / \mathrm{L}$ after $24 \mathrm{~h}$ incubation). However, the derivative 15a did not reach the activity of terbinafine (MIC $0.01-1.72 \mu \mathrm{mol} / \mathrm{L}$ ), that is usually used for therapy of dermatomycosis. 4-Fluoro, 2-bromo or 4-bromo substitution (14a, 17a and 18a) had also inhibiting effect on growth of T. interdigitale (MIC 3.9-7.81 $\mu \mathrm{mol} / \mathrm{L}$ after $24 \mathrm{~h}$ incubation). Halogenated derivatives suppressed the growth of Candida spp. as well. In case of 4-fluoro derivative (14a), it was a moderate, non-specific inhibition (MIC 31.25-62.5 $\mu \mathrm{mol} / \mathrm{L}$ after $24 \mathrm{~h}$ incubation), whereas chlorinated derivatives 15a and 16a inhibited specifically growth of Candida glabrata 20/I and Candida krusei E 28 (in both cases MIC $7.81 \mu \mathrm{mol} / \mathrm{L}$ after $24 \mathrm{~h}$ incubation). The effect of 15a on C. glabrata (MIC $7.81 \mu \mathrm{mol} / \mathrm{L}$ after $24 \mathrm{~h}$ 
incubation) is better than the effect of clinically used antimycotics listed in Table 2. Comparison with previously prepared substances (19-22) is provided as well. Upper mentioned active halogenated derivatives were in common more antifungal effective than hydroxy (20a and 20b), nitro (21a and 21b) or methoxy (22a and 22b) derivatives, with the exception of 2-nitro derivative (21a) inhibiting strongly Candida spp. (antifungal activity in Table 2, structures in Figure 3).

Table 2. Comparison of antifungal activity of selected halogenated 3-phenyl-1-pyrazin-2-ylprop-2-en-1ones with ring B-variously substituted analogues and standard antimycotics.

\begin{tabular}{|c|c|c|c|c|c|c|c|c|c|}
\hline \multirow{4}{*}{ Compd. } & \multirow{4}{*}{$\begin{array}{l}\text { Substituent } \\
\text { in the Ring B }\end{array}$} & \multicolumn{8}{|c|}{$\operatorname{MIC}(\mu \mathrm{mol} / \mathrm{L}) *$} \\
\hline & & CA & CT & CK & CG & TA & AF & LC & TI \\
\hline & & $24 \mathrm{~h}$ & $24 \mathrm{~h}$ & $24 \mathrm{~h}$ & $24 \mathrm{~h}$ & $24 \mathrm{~h}$ & $24 \mathrm{~h}$ & $24 \mathrm{~h}$ & $72 \mathrm{~h}$ \\
\hline & & $48 \mathrm{~h}$ & $48 \mathrm{~h}$ & $48 \mathrm{~h}$ & $48 \mathrm{~h}$ & $48 \mathrm{~h}$ & $48 \mathrm{~h}$ & $48 \mathrm{~h}$ & $120 \mathrm{~h}$ \\
\hline \multirow{2}{*}{$13 a$} & \multirow{2}{*}{$2-\mathrm{F}$} & 15.63 & 250 & 15.62 & 62.5 & 125 & 250 & $>500$ & 250 \\
\hline & & 62.5 & $>500$ & 125 & 125 & 125 & $>500$ & $>500$ & 250 \\
\hline \multirow{2}{*}{$14 a$} & \multirow{2}{*}{$4-\mathrm{F}$} & 31.25 & 62.5 & 31.25 & 62.5 & 62.5 & 250 & $>500$ & 7.81 \\
\hline & & 62.5 & 125 & 62.5 & 125 & 250 & $>500$ & $>500$ & 15.62 \\
\hline \multirow{2}{*}{$15 a$} & \multirow{2}{*}{$2-\mathrm{Cl}$} & $>125$ & $>125$ & 31.25 & 7.81 & 62.5 & $>125$ & $>125$ & 3.9 \\
\hline & & $>125$ & $>125$ & 62.5 & 15.62 & 62.5 & $>125$ & $>125$ & 3.9 \\
\hline \multirow{2}{*}{$16 a$} & \multirow{2}{*}{$4-\mathrm{Cl}$} & 125 & 125 & 7.81 & 31.25 & 31.25 & 62.5 & $>125$ & $>125$ \\
\hline & & $>125$ & $>125$ & 15.62 & 62.5 & 125 & $>125$ & $>125$ & $>125$ \\
\hline \multirow{2}{*}{$17 a$} & \multirow{2}{*}{$2-\mathrm{Br}$} & 62.5 & $>125$ & 62.5 & 7.81 & 62.5 & $>125$ & $>125$ & 7.81 \\
\hline & & $>125$ & $>125$ & $>125$ & 31.25 & 125 & $>125$ & $>125$ & 7.81 \\
\hline \multirow{2}{*}{$18 a$} & \multirow{2}{*}{$4-\mathrm{Br}$} & 15.62 & 31.25 & 32.6 & $>125$ & $>125$ & 125 & $>125$ & 3.9 \\
\hline & & 31.25 & 125 & $>125$ & $>125$ & $>125$ & $>125$ & $>125$ & 7.81 \\
\hline \multirow{2}{*}{$19^{a}$} & \multirow{2}{*}{$\mathrm{H}$} & 15.63 & 31.25 & 31.25 & 31.25 & 250 & 125 & 250 & 7.81 \\
\hline & & 31.25 & 62.5 & 62.5 & 62.5 & 250 & 125 & 500 & 15.63 \\
\hline \multirow{2}{*}{$20 a^{b}$} & \multirow{2}{*}{$2-\mathrm{OH}$} & 62.5 & 125 & 125 & 125 & 125 & 125 & 125 & 15.62 \\
\hline & & 62.5 & 250 & 250 & 250 & 250 & 250 & 250 & 31.25 \\
\hline \multirow{2}{*}{$20 b$} & \multirow{2}{*}{$4-\mathrm{OH}$} & $>250^{c}$ & $>250^{c}$ & $>250^{c}$ & $>250^{c}$ & $>250^{c}$ & $>250^{c}$ & $>250^{c}$ & $62.5^{b}$ \\
\hline & & $>250^{c}$ & $>250^{c}$ & $>250^{c}$ & $>250^{c}$ & $>250^{c}$ & $>250^{c}$ & $>250^{c}$ & $125^{b}$ \\
\hline \multirow{2}{*}{$21 a$} & \multirow{2}{*}{$2-\mathrm{NO}_{2}$} & $7.81^{\mathrm{d}}$ & $7.81^{\mathrm{d}}$ & $7.81^{\mathrm{d}}$ & $7.81^{\mathrm{d}}$ & $>125^{d}$ & $31.25^{\mathrm{d}}$ & $>125^{d}$ & $31.25^{\mathrm{e}}$ \\
\hline & & $15.63^{\mathrm{d}}$ & $7.81^{\mathrm{d}}$ & $15.63^{d}$ & $7.81^{\mathrm{d}}$ & $>125^{d}$ & $>125^{\mathrm{d}}$ & $>125^{\mathrm{d}}$ & $31.25^{\mathrm{e}}$ \\
\hline \multirow{2}{*}{$21 b$} & \multirow{2}{*}{$4-\mathrm{NO}_{2}$} & $31.25^{\mathrm{f}}$ & $>62.5^{\mathrm{f}}$ & $31.25^{f}$ & $>62.5^{\mathrm{f}}$ & $>62.5^{\mathrm{f}}$ & $>62.5^{\mathrm{f}}$ & $>62.5^{\mathrm{f}}$ & $15.63^{\mathrm{e}}$ \\
\hline & & $62.5^{f}$ & $>62.5^{\mathrm{f}}$ & $31.25^{\mathrm{f}}$ & $>62.5^{\mathrm{f}}$ & $>62.5^{\mathrm{f}}$ & $>62.5^{\mathrm{f}}$ & $>62.5^{\mathrm{f}}$ & $15.63^{\mathrm{e}}$ \\
\hline \multirow{2}{*}{$22 a^{d}$} & \multirow{2}{*}{$2-\mathrm{OCH}_{3}$} & 31.25 & 31.25 & $>125$ & 31.25 & $>125$ & $>125$ & $>125$ & 31.25 \\
\hline & & 125 & 62.5 & $>125$ & 125 & $>125$ & $>125$ & $>125$ & 125 \\
\hline & & 62.5 & 125 & 125 & 125 & 500 & 62.5 & 500 & 31.25 \\
\hline $22 b^{d}$ & $2-\mathrm{OCH}_{3}$ & 125 & 250 & 250 & 125 & $>500$ & 125 & $>500$ & 62.5 \\
\hline & & 0.24 & $>500$ & 125 & 41.64 & 250 & $>500$ & $>500$ & 6.51 \\
\hline Fluconaz & & 0.24 & $>500$ & 250 & 250 & 500 & $>500$ & $>500$ & 104 \\
\hline & & 0.005 & 125 & 0.65 & 83.58 & 3.26 & 0.49 & 208 & 0.08 \\
\hline Voricona & & 0.007 & 250 & 1.95 & 250 & 14.32 & 1.3 & 250 & 0.12 \\
\hline Terbinafi & & $6.86^{g}$ & $6.86^{\mathrm{g}}$ & $6.86^{\mathrm{g}}$ & $6.86^{g}$ & - & - & - & $0.01-0.86^{g}$ \\
\hline
\end{tabular}

* MIC defined as $\mathrm{IC}_{80}$ for yeasts and yeast-like organisms and $\mathrm{IC}_{50}$ for molds; Notes: CA = Candida albicans ATCC 44859; CT = Candida tropicalis 156; CK = Candida krusei E 28; CG = Candida glabrata 20/I; TA = Trichosporon asahii 1188; $\mathbf{A F}=$ Aspergillus fumigatus 231; LC = Lichtheimia corymbifera 272; TI = Trichophyton interdigitale 445; ${ }^{a}$ ref. [32]; ${ }^{b}$ ref. [31]; ${ }^{\mathrm{c}}$ ref. [33]; ${ }^{\mathrm{d}}$ ref. [34]; ${ }^{\mathrm{e}}$ ref. [4]; ${ }^{\mathrm{f}}$ ref. [35]; ${ }^{\mathrm{g}} \mathrm{IC}_{50}$ after $48 \mathrm{~h}$ of incubation for yeasts and 7 days of incubation for T. interdigitale [36].<smiles>[R]C=CC(=CC(=O)c1cnccn1)c1ccccc1</smiles>

$19 \mathrm{R}=\mathrm{H}, 20 \mathrm{a} \mathrm{R}=2-\mathrm{OH}, 20 \mathrm{~b} \mathrm{R}=4-\mathrm{OH}, 21 \mathrm{a} \mathrm{R}=2-\mathrm{NO}_{2}, 21 \mathrm{~b} \mathrm{R}=4-\mathrm{NO}_{2}, 22 \mathrm{a} \mathrm{R}=2-\mathrm{OCH}_{3}, 22 \mathrm{~b} \mathrm{R}=4-\mathrm{OCH}_{3}$

Figure 3. Structure of pyrazine-based chalcones comprised in Table 2 for comparison of antifungal activity. 


\subsection{Evaluation of In Vitro Antibacterial Activity}

All prepared compounds, including 16a-16f were subjected to antibacterial assay, and only the most active ones are displayed in Table 3. From the panel of tested bacteria, Staphylococcus spp. was the most susceptible. Growth of Staphylococcus aureus CCM 4516/08 and S. aureus H 5996/08 was inhibited by most compounds listed in Table 2 with moderate effect, but growth of Staphylococcus epidermidis H6966/08 was inhibited more significantly by 2-chlorinated derivatives (MIC of 15a: $3.9 \mu \mathrm{mol} / \mathrm{L}$ after $24 \mathrm{~h}$ incubation and MIC of 15b: $7.81 \mu \mathrm{mol} / \mathrm{L}$ after $24 \mathrm{~h}$ incubation) than by standard antibiotics. Comparing the character of halogen substitution, fluorine does not seem to be as important in our series as in diphenylpropenones [37]. In another study, focused on diphenylpropenones, derivatives with 3,5-dibromo or 3,5-bis(trifluoromethyl) substitution were the most active compounds [38]. In agreement with the positive effect of chlorine substitution in our series, the most antifungal and antibacterial substance in a 3-quinolinyl-1-thienyl propenones had so far three chlorine atoms [39]. Polyhalogenation of 3-phenyl-1-pyrrol-2-ylpropenones also positively reflected in their antimicrobial activity [40]. In a thiazole-based series of chalcones, derivatives chlorinated in the ring $B$ exerted also better antibacterial and antifungal activity than unsubstituted derivative or nitro derivatives [41]. However, pyrimidine analogues of chalcones, variable halogenated in position 2 of the ring B, exert no remarkable antibacterial or antifungal activity [42].

Table 3. Comparison of antibacterial activity of selected novel halogenated 3-phenyl-1-pyrazin-2ylprop-2-en-1-ones with standard antibacterial agents.

\begin{tabular}{|c|c|c|c|c|c|c|c|c|c|c|}
\hline \multirow{4}{*}{ Compd. } & \multirow{2}{*}{\multicolumn{2}{|c|}{ Substituent in the Ring }} & \multicolumn{8}{|c|}{$\mathrm{IC}_{95}(\mu \mathrm{mol} / \mathrm{L})$} \\
\hline & & & SA & MRSA & SE & EF & EC & KP & KP-E & PA \\
\hline & \multirow{2}{*}{$\mathbf{A}$} & \multirow{2}{*}{ B } & $24 \mathrm{~h}$ & $24 \mathrm{~h}$ & $24 \mathrm{~h}$ & $24 \mathrm{~h}$ & $24 \mathrm{~h}$ & $24 \mathrm{~h}$ & $24 \mathrm{~h}$ & $24 \mathrm{~h}$ \\
\hline & & & $48 \mathrm{~h}$ & $48 \mathrm{~h}$ & $48 \mathrm{~h}$ & $48 \mathrm{~h}$ & $48 \mathrm{~h}$ & $48 \mathrm{~h}$ & $48 \mathrm{~h}$ & $48 \mathrm{~h}$ \\
\hline \multirow{2}{*}{$13 a$} & \multirow{2}{*}{$\mathrm{H}$} & \multirow{2}{*}{$2-\mathrm{F}$} & 125 & 250 & 31.25 & 500 & $>500$ & $>500$ & $>500$ & $>500$ \\
\hline & & & 250 & 250 & 125 & 500 & $>500$ & $>500$ & $>500$ & $>500$ \\
\hline \multirow[b]{2}{*}{$13 e$} & \multirow{2}{*}{ 5-pro } & \multirow[b]{2}{*}{$2-\mathrm{F}$} & 125 & $>500$ & 31.25 & $>500$ & $>500$ & $>500$ & $>500$ & $>500$ \\
\hline & & & $>500$ & $>500$ & 125 & $>500$ & $>500$ & $>500$ & $>500$ & $>500$ \\
\hline \multirow{2}{*}{$14 a$} & \multirow{2}{*}{$\mathrm{H}$} & \multirow{2}{*}{$4-\mathrm{F}$} & 125 & 125 & 31.25 & $>500$ & $>500$ & $>500$ & $>500$ & $>500$ \\
\hline & & & 125 & 500 & 62.5 & $>500$ & $>500$ & $>500$ & $>500$ & $>500$ \\
\hline \multirow[b]{2}{*}{$15 a$} & \multirow{2}{*}{$\mathrm{H}$} & \multirow{2}{*}{$2-\mathrm{Cl}$} & 31.25 & 62.5 & 3.9 & 62.5 & $>500$ & $>500$ & $>500$ & $>500$ \\
\hline & & & 62.5 & 250 & 15.62 & 250 & $>500$ & $>500$ & $>500$ & $>500$ \\
\hline \multirow{2}{*}{$15 b$} & \multirow{2}{*}{$5-t-b u$} & \multirow{2}{*}{$2-\mathrm{Cl}$} & 125 & $>500$ & 7.81 & 125 & $>500$ & $>500$ & $>500$ & $>500$ \\
\hline & & & 500 & $>500$ & 31.25 & 500 & $>500$ & $>500$ & $>500$ & $>500$ \\
\hline \multirow{2}{*}{$17 a$} & \multirow[b]{2}{*}{$\mathrm{H}$} & \multirow{2}{*}{$2-\mathrm{Br}$} & 31.25 & 62.5 & 62.5 & $>500$ & $>500$ & $>500$ & $>500$ & $>500$ \\
\hline & & & 31.25 & 250 & 250 & $>500$ & $>500$ & $>500$ & $>500$ & $>500$ \\
\hline \multirow{2}{*}{\multicolumn{3}{|c|}{ Neomycin }} & 2.60 & 1.95 & 9.11 & 291.67 & 2.28 & 1.30 & 2.28 & 7.81 \\
\hline & & & 3.25 & 4.23 & 13.02 & 291.67 & 2.28 & 1.30 & 2.28 & 15.62 \\
\hline \multirow{2}{*}{ Bacitracin } & & & 10.41 & 13.02 & 15.62 & 31.25 & \multirow[t]{2}{*}{-} & \multirow{2}{*}{ - } & \multirow[t]{2}{*}{-} & \multirow[t]{2}{*}{ - } \\
\hline & & & 18.23 & 26.04 & 31.25 & 52.08 & & & & \\
\hline \multirow{2}{*}{ Penicillin } & & & 0.57 & 83.33 & 135.42 & 7.81 & 125.00 & 333.33 & \multirow[t]{2}{*}{ - } & \multirow[b]{2}{*}{-} \\
\hline & & & 0.73 & 104.17 & 208.33 & 15.62 & 125.00 & 416.67 & & \\
\hline
\end{tabular}

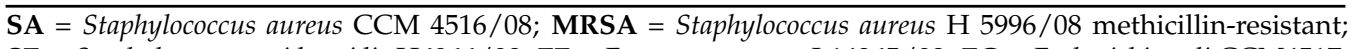
$\mathrm{SE}=$ Staphylococcus epidermidis H6966/08; EF = Enterococcus sp. J 14365/08; EC = Escherichia coli CCM4517; KP = Klebsiella pneumoniae D 11750/08; KP-E = Klebsiella pneumoniae J 14368/08 (ESBL positive, KP-E); PA = Pseudomonas aeruginosa CCM 1961.

\subsection{Evaluation of In Vitro Antimycobacterial Activity}

As far as the antimycobacterial screening is concerned, four strains were tested in total (all results are shown Table 4). The most susceptible strain to inhibition by halogenated pyrazine-based analogues of chalcones seems to be Mycobacterium kansasii Hauduroy CNCTC My 235/80. However, 
the lowest MICs have been achieved in 2-chlorinated compounds (15a and 15b) during testing on Mycobacterium tuberculosis H37RV CNCTC My 331/88 (MIC $6.25 \mu \mathrm{g} / \mathrm{mL}$ for 15a and $3.13 \mu \mathrm{g} / \mathrm{mL}$ for $\mathbf{1 5 b}$ ). The efficacy on $M$. tuberculosis in these two cases correlates well with results of in-house testing on Mycobacterium smegmatis CCM 4622 (ATCC 607) (MIC $7.81 \mu \mathrm{g} / \mathrm{mL}$ for 15a and $3.9 \mu \mathrm{g} / \mathrm{mL}$ for $15 b)$. In comparison with our previous results [4,5,31], positive influence of tert-butyl substitution in the position 5 of ring A has been confirmed again during testing on M. tuberculosis, which is the most pathogenic strain. As for substitution of ring B, 2-halogen substituent seems to be of importance, as products $\mathbf{1 3 a}, \mathbf{1 5 b}$ and $\mathbf{1 7 a}$ showed the lowest MICs (3.13-6.25 $\mu \mathrm{g} / \mathrm{mL}$ against M. tuberculosis H37RV CNCTC My 331/88).

In Table 4, calculated lipophilicities of all tested compounds are provided. They do not seem to correlate with biological activity of the compounds. Conformation of particular molecules influenced by halogen position may play a more important role.

Table 4. Comparison of antimycobacterial activity of halogenated 3-phenyl-1-pyrazin-2-ylprop-2-en1-ones with standard antimycobacterial agents and calculated lipophilicities of the compounds.

\begin{tabular}{|c|c|c|c|c|c|c|c|c|}
\hline \multirow{2}{*}{ Compd. } & \multicolumn{2}{|c|}{ Substituent in the Ring } & \multicolumn{4}{|c|}{ MIC $(\mu \mathrm{g} / \mathrm{mL})$} & \multicolumn{2}{|c|}{ Lipophilicity $^{a}$} \\
\hline & A & B & MK & MA & MT & MS & $\log P$ & $C \log P$ \\
\hline $13 a$ & $\mathrm{H}$ & $2-F$ & 12.5 & 100 & 12.5 & 15.625 & 1.49 & 2.11066 \\
\hline $13 b$ & $5-t-\mathrm{bu}$ & $2-\mathrm{F}$ & 12.5 & $>100$ & 6.25 & $\geq 500$ & 3.62 & 3.93666 \\
\hline $13 e$ & 5-pro & $2-\mathrm{F}$ & 12.5 & $>100$ & 12.5 & 15.625 & 3.1 & 3.66767 \\
\hline $14 a$ & $\mathrm{H}$ & $4-F$ & 12.5 & 100 & 25 & 15.625 & 1.49 & 2.11066 \\
\hline $14 b$ & $5-t-b u$ & $4-F$ & $>25$ & $>25$ & $>25$ & $\geq 125$ & 3.62 & 3.93666 \\
\hline $15 a$ & $\mathrm{H}$ & $2-\mathrm{Cl}$ & 25 & $>100$ & 6.25 & 7.81 & 1.89 & 2.68067 \\
\hline $15 b$ & $5-t-b u$ & $2-\mathrm{Cl}$ & 25 & $>100$ & 3.13 & 3.9 & 4.02 & 4.50666 \\
\hline $16 a$ & $\mathrm{H}$ & 4-Cl & 6.25 & 100 & 50 & 15.625 & 1.89 & 2.68067 \\
\hline $16 b$ & $5-t-\mathrm{bu}$ & $4-\mathrm{Cl}$ & $>50$ & $>50$ & $>50$ & $\geq 250$ & 4.02 & 4.50666 \\
\hline $16 c$ & 5-isobu & $4-\mathrm{Cl}$ & 12.5 & $>25$ & $>25$ & $\geq 125$ & 3.83 & 4.63666 \\
\hline $16 d$ & 5-bu & $4-\mathrm{Cl}$ & 12.5 & 12.5 & $>50$ & $\geq 250$ & 3.92 & 4.76666 \\
\hline $16 e$ & 5-pro & $4-\mathrm{Cl}$ & 12.5 & 12.5 & 25 & $\geq 500$ & 3.50 & 4.23767 \\
\hline $16 f$ & 5-isopro & $4-\mathrm{Cl}$ & 6.25 & $>50$ & 12.5 & $\geq 125$ & 3.48 & 4.10767 \\
\hline $17 a$ & $\mathrm{H}$ & $2-\mathrm{Br}$ & 12.5 & 100 & 25 & 15.625 & 2.16 & 2.83067 \\
\hline $17 b$ & $5-t-\mathrm{bu}$ & $2-\mathrm{Br}$ & 12.5 & 100 & 6.25 & 62.5 & 4.39 & 4.65667 \\
\hline $17 c$ & 5-isobu & $2-\mathrm{Br}$ & 100 & $>100$ & $>100$ & 62.5 & 4.10 & 4.78667 \\
\hline $17 d$ & 5-bu & $2-\mathrm{Br}$ & 25 & $>100$ & 25 & 15.625 & 4.19 & 4.91667 \\
\hline $17 \mathrm{e}$ & 5-pro & $2-\mathrm{Br}$ & 6.25 & $>100$ & 12.5 & $\geq 500$ & 3.77 & 4.38767 \\
\hline $17 f$ & 5-isopro & $2-\mathrm{Br}$ & 12.5 & 50 & 12.5 & 15.625 & 3.75 & 4.25767 \\
\hline $18 a$ & $\mathrm{H}$ & $4-\mathrm{Br}$ & 12.5 & $>100$ & 12.5 & 62.5 & 2.16 & 2.83067 \\
\hline $18 b$ & $5-t-\mathrm{bu}$ & $4-\mathrm{Br}$ & 12.5 & $>50$ & $>50$ & $\geq 250$ & 4.29 & 4.65667 \\
\hline $18 c$ & 5-isobu & $4-\mathrm{Br}$ & 25 & $>100$ & $>100$ & 250 & 4.10 & 4.78667 \\
\hline $18 d$ & $5-b u$ & $4-\mathrm{Br}$ & $>50$ & $>50$ & $>50$ & 125 & 4.19 & 4.91667 \\
\hline $18 \mathrm{e}$ & 5-pro & $4-\mathrm{Br}$ & 12.5 & $>50$ & 50 & $\geq 250$ & 3.77 & 4.38767 \\
\hline $18 f$ & 5-isopro & $4-\mathrm{Br}$ & 6.25 & $>100$ & 25 & $\geq 500$ & 3.75 & 4.25767 \\
\hline $18 g$ & 5-pent & $4-\mathrm{Br}$ & $>50$ & $>50$ & $>50$ & $\geq 250$ & 4.61 & 5.44566 \\
\hline Isoniazid & & & $3.13-12.5$ & $3.13-6.25$ & $0.1-0.39$ & $7.81-15.625$ & & \\
\hline Rifampicin & & & - & - & - & $0.78-1.56$ & & \\
\hline Ciprofloxacin & & & - & - & - & $0.098-0.195$ & & \\
\hline
\end{tabular}

MK = Mycobacterium kansasii Hauduroy CNCTC My 235/80; MA = M. avium ssp. avium Chester CNCTC My 80/72; MT = M. tuberculosis H37RV CNCTC My 331/88; MS = Mycobacterium smegmatis CCM 4622 (ATCC 607); ${ }^{a}$ lipophilicity calculated by ChemDraw Professional 15.0, part of ChemOffice (Perkin Elmer, Waltham, MA, USA).

\section{Experimental Section Materials and Methods}

\subsection{Chemistry}

\subsubsection{Materials and Methods}

Pyrazine-2-carbonitrile, 2-fluorobenzaldehyde, 4-fluorobenzaldehyde, 2-chlorobenzaldehyde, 2-bromobenzaldehyde and 4-bromobenzaldehyde were used as starting compounds and were purchased from Sigma-Aldrich (Prague, Czech Republic). 
5-Alkylated pyrazine-2-carbonitriles and 5-alkylated pyrazin-2-ylethan-1-ones were prepared from pyrazinecarbonitrile according to previously published procedures $[29,30]$ and identity of these intermediates was confirmed by TLC.

Silica gel 0.040-0.063 nm (Merck, Darmstadt, Germany) and Silpearl (Kavalier, Votice, Czech Republic) were used for flash column chromatography. The purity of the products was checked by TLC on aluminium sheets, silica gel $60 \mathrm{~F}_{254}$ (Merck). Mixtures of hexane and ethyl acetate were used for TLC and column chromatography. Analytical samples were dried over anhydrous phosphorus pentoxide under reduced pressure at room temperature. Melting points were determined either on a Boëtius apparatus or on Stuart SMP 20 (Bibby Scientific Ltd., Stone, UK) and are uncorrected. Elemental analyses were performed on an EA 1110 CHNS instrument (CE Instruments, Milano, Italy) or on a Vario Micro Cube Elemental Analyzer (Elementar Analysensysteme GmbH, Hanau, Germany). Infrared spectra were recorded either in $\mathrm{KBr}$ pellets on a Nicolet Impact $400 \mathrm{IR}$ spectrophotometer (Thermo Scientific, Waltham, MA, USA) or on germanium crystal using ATR method (indicated at particular compound spectra) on a Nicolet Impact 6700 IR spectrophotometer (Thermo Scientific). Characteristic wavenumbers are given in $\mathrm{cm}^{-1} .{ }^{1} \mathrm{H}$ - and ${ }^{13} \mathrm{C}-\mathrm{NMR}$ spectra were recorded at ambient temperature on a Varian Mercury-Vx BB 300 spectrometer (Varian Corp., Palo Alto, CA, USA) operating at $300 \mathrm{MHz}$ for ${ }^{1} \mathrm{H}$ and $75 \mathrm{MHz}$ for ${ }^{13} \mathrm{C}$ or on a VNMR S500 $\left(500 \mathrm{MHz}\right.$ for ${ }^{1} \mathrm{H}-\mathrm{NMR}$ a $125 \mathrm{MHz}$ for $\left.{ }^{13} \mathrm{C}-\mathrm{NMR}\right)$. Chemical shifts were recorded as $\delta$ values in $\mathrm{ppm}$, and were indirectly referenced to tetramethylsilane (TMS) via the solvent signal $\left(2.49\right.$ for ${ }^{1} \mathrm{H}, 39.7$ for ${ }^{13} \mathrm{C}$ in DMSO-d6 and 7.26 for ${ }^{1} \mathrm{H}$, 77.0 for ${ }^{13} \mathrm{C}$ in $\mathrm{CDCl}_{3}$ ). Coupling constants $J$ are given in $\mathrm{Hz}$.

\subsubsection{Synthesis of Halogenated (E)-1-(Pyrazin-2-yl)-3-phenylprop-2-en-1-ones}

1-(Pyrazin-2-yl)ethan-1-one or 1-(5-alkylpyrazin-2-yl)ethan-1-one $(0.01 \mathrm{~mol})$ and the corresponding halogenated benzaldehyde $(0.01 \mathrm{~mol})$ were dissolved in pyridine $(4.4 \mathrm{~mL})$. Diethylamine $(0.01 \mathrm{~mol})$ was added, and the reaction mixture was stirred at $80-120^{\circ} \mathrm{C}$ for $1 \mathrm{~h}$. After cooling, the mixture was poured into ice water $(200 \mathrm{~mL})$, acidified to $\mathrm{pH} 3$ with a few drops of acetic acid, and then refrigerated for $24 \mathrm{~h}$. The separation of crude products from water depended on their character. Solids were filtered off and crystallized from anhydrous ethanol, while oily mixtures were extracted with diethyl ether and subjected to flash chromatography on silica gel. Hexane-ethyl acetate was used as the eluent in an appropriate ratio $(70: 30(v / v), 80: 20(v / v)$ or 90:10 $(v / v))$. The fractions containing the desired compounds were combined and crystallized from absolute ethanol to obtain analytically pure crystals. Using this procedure, the following compounds were obtained:

(2E)-3-(2-Fluorophenyl)-1-(pyrazin-2-yl)prop-2-en-1-one (13a). Light yellow powder; yield 18\%, m.p. 110-111 ${ }^{\circ} \mathrm{C}$ (subl.) (114-115 ${ }^{\circ} \mathrm{C}$ [43]); IR (ATR-Ge) 1015, 1056, 1334 (pyrazine) 1597 (C=C), $1670(\mathrm{C}=\mathrm{O})$; ${ }^{1} \mathrm{H}-\mathrm{NMR}\left(300 \mathrm{MHz}, \mathrm{CDCl}_{3}\right) \delta 9.36\left(\mathrm{~d}, 1 \mathrm{H}, J=1.4, \mathrm{H}-3^{\prime}\right), 8.37\left(\mathrm{~d}, 1 \mathrm{H}, J=2.5, \mathrm{H}-6^{\prime}\right), 8.70-8.68(\mathrm{~m}, 1 \mathrm{H}$, H-5 $), 8.23(\mathrm{~d}, 1 \mathrm{H}, J=16.2, \beta-\mathrm{CH}), 8.10(\mathrm{~d}, 1 \mathrm{H}, J=16.2, \alpha-\mathrm{CH}), 7.80-7.70(\mathrm{~m}, 1 \mathrm{H}, \mathrm{H}-6), 7.45-7.35(\mathrm{~m}$, $1 \mathrm{H}, \mathrm{H}-4), 7.24-7.08$ (2H, H3, H-5); ${ }^{13} \mathrm{C}-\mathrm{NMR}\left(75 \mathrm{MHz}, \mathrm{CDCl}_{3}\right) \delta 188.6,161.9(\mathrm{~d}, J=255.1), 148.3,147.5$, $144.9,143.4,138.0(\mathrm{~d}, J=3.2), 132.4(\mathrm{~d}, J=8.6), 129.5(\mathrm{~d}, J=2.6), 124.5(\mathrm{~d}, J=3.7), 122.9(\mathrm{~d}, J=11.4)$, $123.3(\mathrm{~d}, J=6.3), 116.3(\mathrm{~d}, J=22.1)$; elem. anal. calcd. for $\mathrm{C}_{13} \mathrm{H}_{9} \mathrm{FN}_{2} \mathrm{O}(228.23) 68.42 \% \mathrm{C} ; 3.97 \% \mathrm{H}$; $12.27 \% \mathrm{~N}$, found $68.20 \% \mathrm{C} ; 4.09 \% \mathrm{H} ; 12.55 \% \mathrm{~N}$.

(2E)-1-(5-tert-Butylpyrazin-2-yl)-3-(2-fluorophenyl)prop-2-en-1-one (13b). Light yellow powder; yield 13\%; m.p. 97-99 ${ }^{\circ}$ C; IR (ATR-Ge) 1014, 1237, 1282 (pyrazine) $1604(\mathrm{C}=\mathrm{C}), 1671$ (C=O); ${ }^{1} \mathrm{H}-\mathrm{NMR}(500 \mathrm{MHz}$, $\left.\mathrm{CDCl}_{3}\right) \delta 9.27\left(\mathrm{~s}, 1 \mathrm{H}, \mathrm{H}-3^{\prime}\right), 8.73\left(\mathrm{~s}, 1 \mathrm{H}, \mathrm{H}-6^{\prime}\right), 8.23(\mathrm{~d}, 1 \mathrm{H}, J=16.1, \beta-\mathrm{CH}), 8.08(\mathrm{~d}, 1 \mathrm{H}, J=16.1, \alpha-\mathrm{CH})$, $7.74(\mathrm{~d}, 1 \mathrm{H}, J=7.6, \mathrm{H}-6), 7.42-7.35$ (m, 1H, H-4), 7.19 (t, 1H, J = 7.6, H-5), 7.015-7.09 (m, 1H, H-3), 1.44 (s, 9H, $\left.\mathrm{CH}_{3}\right) ;{ }^{13} \mathrm{C}-\mathrm{NMR}\left(125 \mathrm{MHz}, \mathrm{CDCl}_{3}\right) \delta 188.6,167.7,161.9(\mathrm{~d}, J=255.3), 145.6,143.3,139.9,137.4$ $(\mathrm{d}, J=2.9), 132.1(\mathrm{~d}, J=8.9), 129.4(\mathrm{~d}, J=3.0), 124.4(\mathrm{~d}, J=3.9), 123.0(\mathrm{~d}, J=11.8), 122.7(\mathrm{~d}, J=5.8), 116.2$ (d, $J=21.6), 37.1,30.9$; elem. anal. calcd. for $\mathrm{C}_{17} \mathrm{H}_{17} \mathrm{FN}_{2} \mathrm{O}$ (284.33) $71.81 \% \mathrm{C} ; 6.03 \% \mathrm{H} ; 9.85 \% \mathrm{~N}$, found $71.72 \%$ C; $6.36 \% \mathrm{H} ; 10.28 \% \mathrm{~N}$. 
(2E)-3-(2-Fluorophenyl)-1-(5-propylpyrazin-2-yl)prop-2-en-1-one (13e). Light yellow crystals; yield 20\%; m.p. 66-67 ${ }^{\circ}$; IR (ATR-Ge) 1018, 1232, 1321 (pyrazine) 1603 (C=C), 1672 (C=O), ${ }^{1} \mathrm{H}-\mathrm{NMR}(500 \mathrm{MHz}$, $\left.\mathrm{CDCl}_{3}\right) \delta 9.26\left(\mathrm{~s}, 1 \mathrm{H}, \mathrm{H}-3^{\prime}\right), 8.52\left(\mathrm{~s}, 1 \mathrm{H}, \mathrm{H}-6^{\prime}\right), 8.22(\mathrm{~d}, 1 \mathrm{H}, J=16.1, \beta-\mathrm{CH}), 8.08(\mathrm{~d}, 1 \mathrm{H}, J=16.1, \alpha-\mathrm{CH})$, 7.77-7.72 (m, 1H, H-6), 7.41-7.35 (m, 1H, H-4), 7.18 (t, 1H, J = 7.3, H-5), 7.14-7.09 (m, 1H, H-3), 2.88 $\left(\mathrm{t}, 2 \mathrm{H}, \mathrm{J}=7.5, \mathrm{CH}_{2}\right), 1.87-1.76\left(\mathrm{~m}, 2 \mathrm{H}, \mathrm{CH}_{2}\right), 0.99\left(\mathrm{t}, 3 \mathrm{H}, J=7.5, \mathrm{CH}_{3}\right),{ }^{13} \mathrm{C}-\mathrm{NMR}\left(125 \mathrm{MHz}, \mathrm{CDCl}_{3}\right) \delta$ $188.6,161.9(\mathrm{~d}, J=255.1), 161.1,146.0,144.1,142.8,137.5(\mathrm{~d}, J=3.0), 132.2(\mathrm{~d}, J=8.8), 129.4(\mathrm{~d}, J=2.9)$, $124.4(\mathrm{~d}, J=3.9), 123.0(\mathrm{~d}, J=10.8), 122.6(\mathrm{~d}, J=5.9), 116.2(\mathrm{~d}, J=21.5), 37.7,22.5,13.7$; elem. anal. calcd. for $\mathrm{C}_{16} \mathrm{H}_{15} \mathrm{FN}_{2} \mathrm{O}(270.30) 71.10 \% \mathrm{C} ; 5.59 \% \mathrm{H} ; 10.36 \% \mathrm{~N}$, found $71.15 \% \mathrm{C} ; 5.88 \% \mathrm{H} ; 10.89 \% \mathrm{~N}$.

(2E)-3-(4-Fluorophenyl)-1-(pyrazin-2-yl)prop-2-en-1-one (14a). Yellow crystals; yield 6\%; m.p. $120-124{ }^{\circ} \mathrm{C}$; IR (ATR-Ge) 1016, 1058, 1231 (pyrazine), 1589 (C=C), 1671 (C=O), ${ }^{1} \mathrm{H}-\mathrm{NMR}\left(300 \mathrm{MHz}, \mathrm{CDCl}_{3}\right) \delta 9.37$ (s, $\left.1 \mathrm{H}, \mathrm{H}-3^{\prime}\right), 8.77\left(\mathrm{~s}, 1 \mathrm{H}, \mathrm{H}-5^{\prime}\right), 8.68\left(\mathrm{~s}, 1 \mathrm{H}, \mathrm{H}-6^{\prime}\right), 8.11(\mathrm{~d}, 1 \mathrm{H}, J=15.9, \beta-\mathrm{CH}), 7.93(\mathrm{~d}, 1 \mathrm{H}, J=15.9, \alpha-\mathrm{CH})$, 7.78-7.65 (m, 2H, H-2, H-6), 7.11 (t, 2H, J = 8.2, H-3, H-5), ${ }^{13} \mathrm{C}-\mathrm{NMR}\left(75 \mathrm{MHz}, \mathrm{CDCl}_{3}\right) \delta 188.4,164.3$ $(\mathrm{d}, J=252.8), 148.3,147.5,144.8,144.3,143.3,131.0(\mathrm{~d}, J=3.4), 130.9(\mathrm{~d}, J=8.6), 119.7(\mathrm{~d}, J=2.3), 116.2$ $(\mathrm{d}, \mathrm{J}=22.0)$; elem. anal. calcd. for $\mathrm{C}_{13} \mathrm{H}_{9} \mathrm{FN}_{2} \mathrm{O}(228.23) 68.42 \% \mathrm{C} ; 3.97 \% \mathrm{H} ; 12.27 \% \mathrm{~N}$, found $67.98 \% \mathrm{C}$; $4.12 \% \mathrm{H} ; 12.17 \% \mathrm{~N}$.

(2E)-1-(5-tert-Butylpyrazin-2-yl)-3-(4-fluorophenyl)prop-2-en-1-one (14b). Light yellow powder; yield 15\%; m.p. 136-138 ${ }^{\circ} \mathrm{C}$; IR (ATR-Ge) 1011, 1139, 1228 (pyrazine), 1595 (C=C), 1669 (C=O); ${ }^{1} \mathrm{H}-\mathrm{NMR}(300 \mathrm{MHz}$, $\left.\mathrm{CDCl}_{3}\right) \delta 9.28\left(\mathrm{~d}, 1 \mathrm{H}, J=1.5, \mathrm{H}-3^{\prime}\right), 8.73\left(\mathrm{~d}, 1 \mathrm{H}, J=1.5, \mathrm{H}-6^{\prime}\right), 8.10(\mathrm{~d}, 1 \mathrm{H}, J=16.0, \beta-\mathrm{CH}), 7.90(\mathrm{~d}, 1 \mathrm{H}$, $J=16.0, \alpha-\mathrm{CH}), 7.75-7.66(\mathrm{~m}, 2 \mathrm{H}, \mathrm{H}-2, \mathrm{H}-6), 7.11(\mathrm{t}, 2 \mathrm{H}, J=8.7, \mathrm{H}-3, \mathrm{H}-5), 1.45\left(\mathrm{~s}, 9 \mathrm{H}, \mathrm{CH}_{3}\right) ;{ }^{13} \mathrm{C}-\mathrm{NMR}$ (75 MHz, DMSO) $\delta 188.5,167.7,164.2(\mathrm{~d}, J=252.3), 145.6,143.8,143.3,139.8,131.2(\mathrm{~d}, J=3.5), 130.8$ $(\mathrm{d}, J=8.6), 120.1(\mathrm{~d}, J=2.3), 116.1(\mathrm{~d}, J=21.7), 37.1,29.7$; elem. anal. calcd. for. $\mathrm{C}_{17} \mathrm{H}_{17} \mathrm{FN} 2 \mathrm{O}(\mathrm{M} 284.33)$ $71.81 \% \mathrm{C} ; 6.03 \% \mathrm{H} ; 9.85 \% \mathrm{~N}$, found $71.54 \% \mathrm{C} ; 6.22 \% \mathrm{H} ; 10.16 \% \mathrm{~N}$.

(2E)-3-(2-Chlorophenyl)-1-(pyrazin-2-yl)prop-2-en-1-one (15a). Light yellow needles; yield 38\%; m.p. 132.4-134.2 ${ }^{\circ} \mathrm{C}\left(127-129{ }^{\circ} \mathrm{C}\right.$ [43]; IR (ATR-Ge) 1015, 1060, 1330 (pyrazine), 1599 (C=C), 1668 (C=O), ${ }^{1} \mathrm{H}-\mathrm{NMR}\left(500 \mathrm{MHz} \mathrm{CDCl}_{3}\right) \delta 9.39\left(\mathrm{~d}, 1 \mathrm{H}, J=1.5, \mathrm{H}-3^{\prime}\right), 8.79\left(\mathrm{~d}, 1 \mathrm{H}, J=2.5, \mathrm{H}-5^{\prime}\right), 8.71-8.69(\mathrm{~m}, 1 \mathrm{H}$, H-6 $\left.{ }^{\prime}\right), 8.40(\mathrm{~d}, 1 \mathrm{H}, J=16.1, \beta-\mathrm{CH}), 8.17(\mathrm{~d}, 1 \mathrm{H}, J=16.1, \alpha-\mathrm{CH}), 7.88(\mathrm{dd}, 1 \mathrm{H}, J=7.8, J=2.0, \mathrm{H}-6), 7.47-7.44$ (m, 1H, H-3), 7.38-7.30 (m, 2H, H-4, H-5), ${ }^{13} \mathrm{C}-\mathrm{NMR}\left(125 \mathrm{MHz}, \mathrm{CDCl}_{3}\right) \delta 188.3,148.3,147.5,144.9$, $143.3,141.3,136.0,133.0,131.6,130.3,128.0,127.1,122.4$ elem. anal. calcd. for $\mathrm{C}_{13} \mathrm{H}_{9} \mathrm{ClN}_{2} \mathrm{O}(\mathrm{M} 244.68)$ $63.82 \% \mathrm{C} ; 3.71 \% \mathrm{H} ; 11.45 \% \mathrm{~N}$, found $63.76 \% \mathrm{C} ; 3.78 \% \mathrm{H} ; 11.42 \% \mathrm{~N}$.

(2E)-1-(5-tert-Butylpyrazin-2-yl)-3-(2-chlorophenyl)prop-2-en-1-one (15b). Light yellow powder; yield 16\%; m.p. 99.8-101.0 ${ }^{\circ} \mathrm{C}$ IR (ATR-Ge) 1014, 1139, 1272 (pyrazine), 1598 (C=C), $1670(\mathrm{C}=\mathrm{O}) ;{ }^{1} \mathrm{H}-\mathrm{NMR}$ $\left(300 \mathrm{MHz} \mathrm{CDCl}_{3}\right) \delta 9.30\left(\mathrm{~d}, 1 \mathrm{H}, J=1.5, \mathrm{H}-3^{\prime}\right), 8.74\left(\mathrm{~d}, 1 \mathrm{H}, J=1.5, \mathrm{H}-6^{\prime}\right), 8.38(\mathrm{~d}, 1 \mathrm{H}, J=15.9, \beta-\mathrm{CH})$, $8.16(\mathrm{~d}, 1 \mathrm{H}, J=15.9, \alpha-\mathrm{CH}), 7.87(\mathrm{~d}, 1 \mathrm{H}, J=7.3, J=2.0, \mathrm{H}-6), 7.47-7.43(\mathrm{~m}, 1 \mathrm{H}, \mathrm{H}-3), 7.37-7.29(\mathrm{~m}, 2 \mathrm{H}$, H-4, H-5), 1.49 (s, 9H, CH $) ;{ }^{13} \mathrm{C}-\mathrm{NMR}$ (75 MHz, DMSO) $\delta 188.4,167.8,145.6,143.4,140.7,139.8,135.9$, 133.1, 131.4, 130.3, 128.0, 127.0, 122.8, 37.1, 29.7; elem. anal. calcd for $\mathrm{C}_{17} \mathrm{H}_{17} \mathrm{ClN}_{2} \mathrm{O}(300.79) 67.88 \%$; $5.70 \% \mathrm{H} ; 9.31 \% \mathrm{~N}$, found $68.18 \% \mathrm{C} ; 5.65 \% \mathrm{H} ; 9.42 \% \mathrm{~N}$.

(2E)-3-(2-Bromophenyl)-1-pyrazin-2-yl)prop-2-en-1-one (17a). Light yellow crystals; yield 50\%; m.p. 130.0-133.0 ${ }^{\circ} \mathrm{C}\left(128-130{ }^{\circ} \mathrm{C}\right.$ [43]); IR (KBr) 1016, 1029, 1059, 1330 (pyrazine) 1601 (C=C), 1665 (C=O); ${ }^{1} \mathrm{H}-\mathrm{NMR}\left(300 \mathrm{MHz}, \mathrm{CDCl}_{3}\right) \delta 9.38\left(1 \mathrm{H}, \mathrm{d}, J=1.5, \mathrm{H}-3^{\prime}\right), 8.78\left(1 \mathrm{H}, \mathrm{d}, J=2.5, \mathrm{H}-5^{\prime}\right), 8.69(1 \mathrm{H}, \mathrm{dd}, J=2.5$, $\left.J=1.5, \mathrm{H}-6^{\prime}\right), 8.35(1 \mathrm{H}, \mathrm{d}, J=16.1, \beta-\mathrm{H}), 8.11(1 \mathrm{H}, \mathrm{d}, J=16.1, \alpha-\mathrm{H}), 7.85(1 \mathrm{H}, \mathrm{dd}, J=8.0, J=1.6, \mathrm{H}-3)$, $7.64(1 \mathrm{H}, \mathrm{dd}, J=8.0, J=1.6, \mathrm{H}-6), 7.40-7.33(1 \mathrm{H}, \mathrm{m}, \mathrm{H}-4), 7.30-7.22(1 \mathrm{H}, \mathrm{m}, \mathrm{H}-5) ;{ }^{13} \mathrm{C}-\mathrm{NMR}(75 \mathrm{MHz}$, $\left.\mathrm{CDCl}_{3}\right) \delta 188.2,148.2,147.6,144.9,143.9,143.3,134.7,133.6,131.7,128.1,127.7,126.5,122.6$; elem. anal. calcd. for $\mathrm{C}_{13} \mathrm{H}_{9} \mathrm{BrN}_{2} \mathrm{O}$ (M 289.13) 54.00\% C; 3.14\% H; 9.69\% N, found $53.59 \%$ C; 3.27\% H; 9.74\% N.

(2E)-3-(2-Bromophenyl)-1-(5-tert-butylpyrazin-2-yl)prop-2-en-1-one (17b). Light yellow crystals; yield 40\%; m.p. 91.0-94.5 ${ }^{\circ} \mathrm{C}$; IR (KBr) 1015, 1040, 1316 (pyrazine), 1597 (C=C), 1669 (C=O); ${ }^{1} \mathrm{H}-\mathrm{NMR}(300 \mathrm{MHz}$, $\left.\mathrm{CDCl}_{3}\right) \delta 9.29\left(1 \mathrm{H}, \mathrm{d}, J=1.5, \mathrm{H}-3^{\prime}\right), 8.73\left(1 \mathrm{H}, \mathrm{d}, J=1.5, \mathrm{H}-6^{\prime}\right), 8.33(1 \mathrm{H}, \mathrm{d}, J=16.1, \beta-\mathrm{H}), 8.11(1 \mathrm{H}, \mathrm{d}$, $J=16.1, \alpha-\mathrm{H}), 7.85(1 \mathrm{H}, \mathrm{dd}, J=7.7, J=1.5, \mathrm{H}-3), 7.64(1 \mathrm{H}, \mathrm{dd}, J=7.7, J=1.5, \mathrm{H}-6), 7.40-7.32(1 \mathrm{H}, \mathrm{m}$, $\mathrm{H}-4), 7.25(1 \mathrm{H}, \mathrm{td}, J=7.7, J=1.5, \mathrm{H}-5), 1.45\left(9 \mathrm{H}, \mathrm{s}, \mathrm{CH}_{3}\right) ;{ }^{13} \mathrm{C}-\mathrm{NMR}\left(75 \mathrm{MHz}^{\mathrm{CDCl}}\right)_{3} \delta 188.3,167.8$, 
145.6, 143.4, 143.3, 139.8, 134.9, 133.6, 131.6, 128.1, 127.7, 126.4, 123.0, 37.1, 29.7; elem. anal. calcd. for $\mathrm{C}_{17} \mathrm{H}_{17} \mathrm{BrN}_{2} \mathrm{O}(345.23) 59.14 \% \mathrm{C} ; 4.96 \% \mathrm{H} ; 8.11 \% \mathrm{~N}$, found $59.05 \% \mathrm{C} ; 5.13 \% \mathrm{H} ; 8.21 \% \mathrm{~N}$.

(2E)-3-(2-Bromophenyl)-1-(5-isobutylpyrazin-2-yl)prop-2-en-1-one (17c). Light yellow crystals; yield 29\%; m.p. 96.0-100.0 ${ }^{\circ} \mathrm{C}$; IR (KBr) 1019, 1049, 1309 (pyrazine), 1598 (C=C), 1698 (C=O); ${ }^{1} \mathrm{H}-\mathrm{NMR}(300 \mathrm{MHz}$, $\left.\mathrm{CDCl}_{3}\right) \delta 9.29\left(1 \mathrm{H}, \mathrm{d}, J=1.4, \mathrm{H}-3^{\prime}\right), 8.49\left(1 \mathrm{H}, \mathrm{d}, J=1.4, \mathrm{H}^{\prime} 6^{\prime}\right), 8.33(1 \mathrm{H}, \mathrm{d}, J=15.9, \beta-\mathrm{H}), 8.11(1 \mathrm{H}, \mathrm{d}$, $J=15.9, \alpha-\mathrm{H}), 7.85(1 \mathrm{H}, \mathrm{dd}, J=7.9, J=1.7, \mathrm{H}-3), 7.64(1 \mathrm{H}, \mathrm{dd}, J=7.9, J=1.7, \mathrm{H}-6), 7.40-7.31(1 \mathrm{H}, \mathrm{m}$, $\mathrm{H}-4), 7.25(1 \mathrm{H}, \mathrm{td}, J=7.9, J=1.7, \mathrm{H}-5), 2.78\left(2 \mathrm{H}, \mathrm{d}, J=6.9, \mathrm{CH}_{2}\right), 2.27-2.08(1 \mathrm{H}, \mathrm{m}, \mathrm{CH}), 0.97(6 \mathrm{H}, \mathrm{d}$, $\left.J=6.9, \mathrm{CH}_{3}\right) ;{ }^{13} \mathrm{C}-\mathrm{NMR}\left(75 \mathrm{MHz}, \mathrm{CDCl}_{3}\right) \delta 188.3,160.6,145.9,144.2,143.4,143.3,134.9,133.6,131.6$, 128.2, 127.6, 126.4, 123.0, 44.8, 29.1, 22.3; elem. anal. calcd. for $\mathrm{C}_{17} \mathrm{H}_{17} \mathrm{BrN}_{2} \mathrm{O}$ (M 345.24): 59.14\% C; $4.96 \% \mathrm{H} ; 8.11 \% \mathrm{~N}$, found $59.31 \% \mathrm{C} ; 5.02 \% \mathrm{H} ; 8.42 \% \mathrm{~N}$.

(2E)-3-(2-Bromophenyl)-1-(5-butylpyrazin-2-yl)prop-2-en-1-one (17d). Light yellow crystals; yield 20\%; m.p. 83.0-88.0 ${ }^{\circ} \mathrm{C}$; IR (KBr) 1019, 1051, 1320 (pyrazine), $1602(\mathrm{C}=\mathrm{C}), 1674(\mathrm{C}=\mathrm{O}) ;{ }^{1} \mathrm{H}-\mathrm{NMR}(300 \mathrm{MHz}$, $\left.\mathrm{CDCl}_{3}\right) \delta 9.28\left(1 \mathrm{H}, \mathrm{d}, J=1.2, \mathrm{H}-3^{\prime}\right), 8.52\left(1 \mathrm{H}, \mathrm{d}, J=1.2, \mathrm{H}-6^{\prime}\right), 8.33(1 \mathrm{H}, \mathrm{d}, J=15.8, \beta-\mathrm{H}), 8.10(1 \mathrm{H}, \mathrm{d}$, $J=15.8, \alpha-\mathrm{H}), 7.85(1 \mathrm{H}, \mathrm{dd}, J=7.6, J=1.3, \mathrm{H}-3), 7.63(1 \mathrm{H}, \mathrm{dd}, J=7.6, J=1.3, \mathrm{H}-6), 7.39-7.32(1 \mathrm{H}, \mathrm{m}$, $\mathrm{H}-4), 7.25(1 \mathrm{H}, \mathrm{dt}, J=7.6, J=1.3, \mathrm{H}-5), 2.91\left(2 \mathrm{H}, \mathrm{t}, J=7.6, \mathrm{CH}_{2}\right), 1.85-1.69\left(2 \mathrm{H}, \mathrm{m}, \mathrm{CH}_{2}\right), 1.50-1.33(2 \mathrm{H}$, $\left.\mathrm{m}, \mathrm{CH}_{2}\right), 0.96\left(3 \mathrm{H}, \mathrm{t}, J=7.6, \mathrm{CH}_{3}\right) ;{ }^{13} \mathrm{C}-\mathrm{NMR}\left(75 \mathrm{MHz}, \mathrm{CDCl}_{3}\right) \delta 188.3,161.5,145.9,144.1,143.4,142.8$, $134.9,133.5,131.6,128.1,127.6,126.4,123.0,35.5,31.4,22.4,13.8$; elem. anal. calcd. for $\mathrm{C}_{17} \mathrm{H}_{17} \mathrm{BrN}_{2} \mathrm{O}$ (M 345.24) $59.14 \% \mathrm{C} ; 4.96 \% \mathrm{H} ; 8.11 \% \mathrm{~N}$, found $59.20 \% \mathrm{C} ; 5.34 \% \mathrm{H} ; 8.29 \% \mathrm{~N}$.

(2E)-3-(2-Bromophenyl)-1-(5-propylpyrazin-2-yl)prop-2-en-1-one (17e). Light yellow crystals; yield 24\%; m.p. 105.0-107.0 ${ }^{\circ} \mathrm{C}$; IR (KBr) 1018, 1059, 1317 (pyrazine) 1603 (C=C), $1672(\mathrm{C}=\mathrm{O}) ;{ }^{1} \mathrm{H}-\mathrm{NMR}(300 \mathrm{MHz}$, $\left.\mathrm{CDCl}_{3}\right) \delta 9.28\left(1 \mathrm{H}, \mathrm{d}, J=1.4, \mathrm{H}-3^{\prime}\right), 8.52\left(1 \mathrm{H}, \mathrm{d}, J=1.4, \mathrm{H}^{\prime} 6^{\prime}\right), 8.32(1 \mathrm{H}, \mathrm{d}, J=15.9, \beta-\mathrm{H}), 8.10(1 \mathrm{H}, \mathrm{d}$, $J=15.9, \alpha-\mathrm{H}), 7.85(1 \mathrm{H}, \mathrm{dd}, J=7.8, J=1.6, \mathrm{H}-3), 7.63(1 \mathrm{H}, \mathrm{dd}, J=7.8, J=1.6, \mathrm{H}-6), 7.39-7.32(1 \mathrm{H}, \mathrm{m}$, $\left.\mathrm{H}-4^{\prime \prime}\right), 7.25(1 \mathrm{H}, \mathrm{td}, J=7.8, J=1.6, \mathrm{H}-5), 2.89\left(2 \mathrm{H}, \mathrm{t}, J=7.4, \mathrm{CH}_{2}\right), 1.91-1.74\left(2 \mathrm{H}, \mathrm{m}, \mathrm{CH}_{2}\right), 1.00(3 \mathrm{H}, \mathrm{d}$, $\left.J=7.4, \mathrm{CH}_{3}\right) ;{ }^{13} \mathrm{C}-\mathrm{NMR}\left(75 \mathrm{MHz}, \mathrm{CDCl}_{3}\right) \delta 188.3,161.2,146.0,144.1,143.4,142.8,134.9,133.5,131.6$, 128.1, 127.6, 126.4, 123.0, 37.7, 22.6, 13.8; elem. anal. calcd. for $\mathrm{C}_{16} \mathrm{H}_{15} \mathrm{BrN}_{2} \mathrm{O}$ (M 331.21) 58.02\% C; $4.57 \% \mathrm{H} ; 8.46 \% \mathrm{~N}$, found $58.01 \% \mathrm{C} ; 4.70 \% \mathrm{H} ; 8.49 \% \mathrm{~N}$.

(2E)-3-(2-Bromophenyl)-1-(5-isopropylpyrazin-2-yl)prop-2-en-1-one (17f). Light yellow needles; yield 33\%; m.p. 71.0-73.0 ${ }^{\circ} \mathrm{C}$; IR (KBr) 1016, 1313 (pyrazine) $1602(\mathrm{C}=\mathrm{C}), 1668(\mathrm{C}=\mathrm{O}) ;{ }^{1} \mathrm{H}-\mathrm{NMR}\left(300 \mathrm{MHz}, \mathrm{CDCl}_{3}\right)$ $\delta 9.29\left(1 \mathrm{H}, \mathrm{d}, J=1.4, \mathrm{H}-3^{\prime}\right), 8.55\left(1 \mathrm{H}, \mathrm{d}, J=1.4, \mathrm{H}-6^{\prime}\right), 8.33(1 \mathrm{H}, \mathrm{d}, J=15.9, \beta-\mathrm{H}), 8.11(1 \mathrm{H}, \mathrm{d}, J=15.9$, $\alpha-\mathrm{H}), 7.85(1 \mathrm{H}, \mathrm{dd}, J=7.8, J=1.6, \mathrm{H}-3), 7.64(1 \mathrm{H}, \mathrm{dd}, J=7.8, J=1.6, \mathrm{H}-6), 7.39-7.32\left(1 \mathrm{H}, \mathrm{m}, \mathrm{H}-4^{\prime \prime}\right)$, $7.25(1 \mathrm{H}, \mathrm{td}, J=7.8, J=1.6, \mathrm{H}-5), 3.32-3.12(1 \mathrm{H}, \mathrm{m}, \mathrm{CH}), 1.38\left(6 \mathrm{H}, \mathrm{d}, J=6.9, \mathrm{CH}_{3}\right) ;{ }^{13} \mathrm{C}-\mathrm{NMR}(75 \mathrm{MHz}$, $\left.\mathrm{CDCl}_{3}\right) \delta 188.3,165.8,146.1,144.0,143.4,141.5,134.9,133.6,131.6,128.1,127.7,126.4,123.0,34.4,22.0$; elem. anal. calcd. for $\mathrm{C}_{16} \mathrm{H}_{15} \mathrm{BrN}_{2} \mathrm{O}(\mathrm{M} 331.21) 58.02 \% \mathrm{C} ; 4.57 \% \mathrm{H} ; 8.46 \% \mathrm{~N}$, found $58.11 \% \mathrm{C} ; 4.57 \% \mathrm{H}$; $8.81 \% \mathrm{~N}$.

(2E)-3-(4-Bromophenyl)-1-(pyrazin-2-yl)prop-2-en-1-one (18a). Light yellow crystals; yield 5\%; m.p. 163.0-168.0 ${ }^{\circ} \mathrm{C}$; IR (KBr) 1017, 1059, 1332 (pyrazine) 1605 (C=C), 1671 (C=O); ${ }^{1} \mathrm{H}-\mathrm{NMR}(300 \mathrm{MHz}$, $\left.\mathrm{CDCl}_{3}\right) \delta 9.37\left(1 \mathrm{H}, \mathrm{d}, J=1.5, \mathrm{H}-3^{\prime}\right), 8.78\left(1 \mathrm{H}, \mathrm{d}, J=2.5, \mathrm{H}-6^{\prime}\right), 8.69\left(1 \mathrm{H}, \mathrm{dd}, J=2.5, J=1.5, \mathrm{H}-5^{\prime}\right), 8.17(1 \mathrm{H}$, $\mathrm{d}, J=16.1, \beta-\mathrm{H}), 7.89(1 \mathrm{H}, \mathrm{d}, J=16.1, \alpha-\mathrm{H}), 7.61-7.52(4 \mathrm{H}, \mathrm{m}, \mathrm{H}-2, \mathrm{H}-3, \mathrm{H}-5, \mathrm{H}-6) ;{ }^{13} \mathrm{C}-\mathrm{NMR}(75 \mathrm{MHz}$, $\left.\mathrm{CDCl}_{3}\right) \delta 188.4,148.2,147.6,144.9,144.2,143.3,133.6,132.2,130.2,125.3,120.5 ;$ elem. anal. calcd for $\mathrm{C}_{13} \mathrm{H}_{9} \mathrm{BrN}_{2} \mathrm{O}(289.13) 54.00 \% \mathrm{C} ; 3.14 \% \mathrm{H} ; 9.69 \% \mathrm{~N}$, found $53.19 \% \mathrm{C} ; 3.58 \% \mathrm{H} ; 9.21 \% \mathrm{~N}$.

(2E)-3-(4-Bromophenyl)-1-(5-tert-butylpyrazin-2-yl)prop-2-en-1-one (18b). Light yellow powder; yield 2\%; m.p. 139.0-142.0 ${ }^{\circ} \mathrm{C}$; IR (KBr) 1020, 1041, 1302 (pyrazine), 1603 (C=C), 1669 (C=O); ${ }^{1} \mathrm{H}-\mathrm{NMR}(300 \mathrm{MHz}$, $\left.\mathrm{CDCl}_{3}\right) \delta 9.28\left(1 \mathrm{H}, \mathrm{d}, J=1.4, \mathrm{H}-3^{\prime}\right), 8.73\left(1 \mathrm{H}, \mathrm{d}, J=1.4, \mathrm{H}-6^{\prime}\right), 8.17(1 \mathrm{H}, \mathrm{d}, J=16.1, \beta-\mathrm{H}), 7.87(1 \mathrm{H}, \mathrm{d}$, $J=16.1, \alpha-\mathrm{H}), 7.62-7.51(4 \mathrm{H}, \mathrm{m}, \mathrm{H}-2, \mathrm{H}-3, \mathrm{H}-5, \mathrm{H}-6), 1.45\left(9 \mathrm{H}, \mathrm{s}, \mathrm{CH}_{3}\right) ;{ }^{13} \mathrm{C}-\mathrm{NMR}\left(75 \mathrm{MHz}, \mathrm{CDCl}_{3}\right) \delta$ 188.5, 167.8, 145.6, 143.6, 143.3, 139.9, 133.8, 132.2, 130.2, 125.1, 120.9, 37.1, 29.7; elem. anal. calcd for $\mathrm{C}_{17} \mathrm{H}_{17} \mathrm{BrN}_{2} \mathrm{O}(345.24) 59.14 \% \mathrm{C} ; 4.96 \% \mathrm{H} ; 8.11 \% \mathrm{~N}$, found $59.34 \% \mathrm{C} ; 5.14 \% \mathrm{H} ; 8.24 \% \mathrm{~N}$. 
(2E)-3-(4-Bromophenyl)-1-(5-isobutylpyrazin-2-yl)prop-2-en-1-one (18c). Light yellow needles; yield 3\%; m.p. 109.0-111.0 ${ }^{\circ} \mathrm{C}$; IR (KBr) 1020, 1043, 1304 (pyrazine) $1602(\mathrm{C}=\mathrm{C}), 1668(\mathrm{C}=\mathrm{O}) ;{ }^{1} \mathrm{H}-\mathrm{NMR}(300 \mathrm{MHz}$, $\left.\mathrm{CDCl}_{3}\right) \delta 9.28\left(1 \mathrm{H}, \mathrm{d}, J=1.4, \mathrm{H}-3^{\prime}\right), 8.49\left(1 \mathrm{H}, \mathrm{d}, J=1.4, \mathrm{H}-6^{\prime}\right), 8.17(1 \mathrm{H}, \mathrm{d}, J=16.1, \beta-\mathrm{H}), 7.87(1 \mathrm{H}, \mathrm{d}$, $J=16.1, \alpha-\mathrm{H}), 7.61-7.51(4 \mathrm{H}, \mathrm{m}, \mathrm{H}-2, \mathrm{H}-3, \mathrm{H}-5, \mathrm{H}-6), 2.79\left(2 \mathrm{H}, \mathrm{d}, J=6.9, \mathrm{CH}_{2}\right), 2.27-2.08(1 \mathrm{H}, \mathrm{m}, \mathrm{CH})$, $0.97\left(6 \mathrm{H}, \mathrm{d}, J=6.9, \mathrm{CH}_{3}\right) ;{ }^{13} \mathrm{C}-\mathrm{NMR}\left(75 \mathrm{MHz} \mathrm{CDCl}_{3}\right) \delta 188.4,160.6,146.0,144.0,143.8,143.3,133.7$, 132.2, 130.2, 125.2, 120.8, 44.7, 29.2, 22.3; elem. anal. calcd. for $\mathrm{C}_{17} \mathrm{H}_{17} \mathrm{BrN}_{2} \mathrm{O}$ (345.24) 59.14\% C; $4.96 \%$ $\mathrm{H} ; 8.11 \% \mathrm{~N}$, found $58.96 \% \mathrm{C} ; 5.21 \% \mathrm{H} ; 8.21 \% \mathrm{~N}$.

(2E)-3-(4-Bromophenyl)-1-(5-butylpyrazin-2-yl)prop-2-en-1-one (18d). Light yellow crystals; yield 2\%; m.p. 115.0-116.0 ${ }^{\circ} \mathrm{C}$; IR (KBr) 1009, 1071, 1330 (pyrazine), 1600 (C=C), 1669 (C=O); ${ }^{1} \mathrm{H}-\mathrm{NMR}(300 \mathrm{MHz}$, $\left.\mathrm{CDCl}_{3}\right) \delta 9.27\left(1 \mathrm{H}, \mathrm{d}, J=1.4, \mathrm{H}-3^{\prime}\right), 8.52\left(1 \mathrm{H}, \mathrm{d}, J=1.4, \mathrm{H}-6^{\prime}\right), 8.16(1 \mathrm{H}, \mathrm{d}, J=16.2, \beta-\mathrm{H}), 7.87(1 \mathrm{H}, \mathrm{d}$, $J=16.2, \alpha-\mathrm{H}), 7.62-7.51(4 \mathrm{H}, \mathrm{m}, \mathrm{H}-2, \mathrm{H}-3, \mathrm{H}-5, \mathrm{H}-6), 2.92\left(2 \mathrm{H}, \mathrm{t}, J=7.5, \mathrm{CH}_{2}\right), 1.85-1.70\left(2 \mathrm{H}, \mathrm{m}, \mathrm{CH}_{2}\right)$, 1.50-1.34 (2H, m, CH 2$), 0.96\left(3 \mathrm{H}, \mathrm{t}, J=7.5, \mathrm{CH}_{3}\right) ;{ }^{13} \mathrm{C}-\mathrm{NMR}\left(75 \mathrm{MHz}, \mathrm{CDCl}_{3}\right) \delta$ 188.4, 161.5, 145.9, 144.1, 143.7, 142.8, 133.8, 132.2, 130.2, 125.1, 120.9, 35.5, 31.4, 22.4, 13.82; elem. anal. calcd. for $\mathrm{C}_{17} \mathrm{H}_{17} \mathrm{BrN}_{2} \mathrm{O}$ (345.24) $59.14 \%$ C; $4.96 \% \mathrm{H} ; 8.11 \% \mathrm{~N}$, found $59.05 \% \mathrm{C} ; 5.33 \% \mathrm{H} ; 8.40 \% \mathrm{~N}$.

(2E)-3-(4-Bromophenyl)-1-(5-propylpyrazin-2-yl)prop-2-en-1-one (18e). Light yellow crystals; yield 4\%; m.p. 99.0-100.0 ${ }^{\circ} \mathrm{C}$; IR (KBr) 1009, 1045, 1071, 1321 (pyrazine) 1600 (C=C), 1670 (C=O); ${ }^{1} \mathrm{H}-\mathrm{NMR}(300 \mathrm{MHz}$, $\left.\mathrm{CDCl}_{3}\right) \delta 9.27\left(1 \mathrm{H}, \mathrm{d}, J=1.4, \mathrm{H}-3^{\prime}\right), 8.52\left(1 \mathrm{H}, \mathrm{d}, J=1.4, \mathrm{H}-6^{\prime}\right), 8.16(1 \mathrm{H}, \mathrm{d}, J=15.9, \beta-\mathrm{H}), 7.87(1 \mathrm{H}, \mathrm{d}$, $J=15.9, \alpha-\mathrm{H}), 7.61-7.51(4 \mathrm{H}, \mathrm{m}, \mathrm{H}-2, \mathrm{H}-3, \mathrm{H}-5, \mathrm{H}-6), 2.90\left(2 \mathrm{H}, \mathrm{d}, J=7.6, \mathrm{CH}_{2}\right), 1.91-1.75\left(1 \mathrm{H}, \mathrm{m}, \mathrm{CH}_{2}\right)$, $1.01\left(3 \mathrm{H}, \mathrm{t}, \mathrm{J}=7.6, \mathrm{CH}_{3}\right) ;{ }^{13} \mathrm{C}-\mathrm{NMR}\left(75 \mathrm{MHz} \mathrm{CDCl}_{3}\right) \delta 188.4,161.2,146.0,144.0,143.7,142.9,133.8$, 132.2, 130.2, 125.1, 120.8, 37.7, 22.6, 13.8; elem. anal. calcd. for $\mathrm{C}_{16} \mathrm{H}_{15} \mathrm{BrN}_{2} \mathrm{O}$ (331.21) 58.02\% C; $4.57 \%$ $\mathrm{H} ; 8.46 \% \mathrm{~N}$, found $57.63 \% \mathrm{C} ; 4.80 \% \mathrm{H} ; 8.57 \% \mathrm{~N}$.

(2E)-3-(4-Bromophenyl)-1-(5-isopropylpyrazin-2-yl)prop-2-en-1-one (18f). Light yellow crystals; yield 4\%; m.p. 106.0-108.0 ${ }^{\circ} \mathrm{C}$; IR (KBr) 1017, 1040, 1068, 1334 (pyrazine) $1600(\mathrm{C}=\mathrm{C}), 1667(\mathrm{C}=\mathrm{O}) ;{ }^{1} \mathrm{H}-\mathrm{NMR}$ $\left(300 \mathrm{MHz}, \mathrm{CDCl}_{3}\right) \delta 9.27\left(1 \mathrm{H}, \mathrm{d}, J=1.5, \mathrm{H}-3^{\prime}\right), 8.56\left(1 \mathrm{H}, \mathrm{d}, J=1.5, \mathrm{H}-6^{\prime}\right), 8.16(1 \mathrm{H}, \mathrm{d}, J=15.8, \beta-\mathrm{H})$, $7.87(1 \mathrm{H}, \mathrm{d}, J=15.8, \alpha-\mathrm{H}), 7.61-7.52(4 \mathrm{H}, \mathrm{m}, \mathrm{H}-2, \mathrm{H}-3, \mathrm{H}-5, \mathrm{H}-6), 3.31-3.15(1 \mathrm{H}, \mathrm{m}, \mathrm{CH}), 1.38(6 \mathrm{H}, \mathrm{d}$, $\left.J=7.0, \mathrm{CH}_{3}\right) ;{ }^{13} \mathrm{C}-\mathrm{NMR}\left(75 \mathrm{MHz}_{,} \mathrm{CDCl}_{3}\right) \delta 188.4,165.8,146.2,143.9,143.7,141.5,133.8,132.2,130.2$, 125.1, 120.9, 34.3, 22.0; elem. anal. calcd. for $\mathrm{C}_{16} \mathrm{H}_{15} \mathrm{BrN}_{2} \mathrm{O}(331.21) 58.02 \% \mathrm{C} ; 4.57 \% \mathrm{H} ; 8.46 \% \mathrm{~N}$, found $57.92 \%$ C; $4.75 \% \mathrm{H} ; 8.52 \% \mathrm{~N}$.

(2E)-3-(4-Bromophenyl)-1-(5-pentylpyrazin-2-yl)prop-2-en-1-one (18g). Light yellow needles; yield 6\%; m.p. 98.0-100.0 ${ }^{\circ} \mathrm{C}$; IR (KBr) 1010, 1036, 1075, 1320 (pyrazine) 1603 (C=C), 1669 (C=O); ${ }^{1} \mathrm{H}-\mathrm{NMR}(300 \mathrm{MHz}$, $\left.\mathrm{CDCl}_{3}\right) \delta 9.27\left(1 \mathrm{H}, \mathrm{d}, J=1.1, \mathrm{H}-3^{\prime}\right), 8.52\left(1 \mathrm{H}, \mathrm{d}, J=1.1, \mathrm{H}-6^{\prime}\right), 8.16(1 \mathrm{H}, \mathrm{d}, J=16.2, \beta-\mathrm{H}), 7.87(1 \mathrm{H}, \mathrm{d}$, $J=16.2 \mathrm{~Hz}, \alpha-\mathrm{H}), 7.62-7.51(4 \mathrm{H}, \mathrm{m}, \mathrm{H}-2, \mathrm{H}-3, \mathrm{H}-5, \mathrm{H}-6), 2.91\left(2 \mathrm{H}, \mathrm{t}, J=7.4, \mathrm{CH}_{2}\right), 1.87-1.71(2 \mathrm{H}, \mathrm{m}$, $\left.\mathrm{CH}_{2}\right), 1.46-1.28\left(4 \mathrm{H}, \mathrm{m}, \mathrm{CH}_{2}\right), 0.90\left(3 \mathrm{H}, \mathrm{t}, \mathrm{J}=7.4, \mathrm{CH}_{3}\right) ;{ }^{13} \mathrm{C}-\mathrm{NMR}\left(75 \mathrm{MHz}, \mathrm{CDCl}_{3}\right) \delta 188.4,161.5$, 145.9, 144.1, 143.7, 142.8, 133.8, 132.2, 130.2, 125.1, 120.9, 35.8, 31.4, 29.0, 22.4, 13.9; elem. anal. calcd. for $\mathrm{C}_{18} \mathrm{H}_{19} \mathrm{BrN}_{2} \mathrm{O}$ (359.27) 60.18\% C; $5.33 \% \mathrm{H} ; 7.80 \% \mathrm{~N}$, found $59.72 \% \mathrm{C} ; 5.58 \% \mathrm{H} ; 7.88 \% \mathrm{~N}$.

\subsection{Biological Evaluation}

\subsubsection{Evaluation of In Vitro Antifungal Activity}

The antifungal activity of all compounds was evaluated by the modified microdilution broth CSLI standards [44,45]. The organisms examined included Candida albicans ATCC 44859 (American Type Culture Collection, Manassas, VA, USA), Candida tropicalis 156, Candida krusei E 28, Candida glabrata 20/I, Trichosporon asahii 1188, Aspergillus fumigatus 231, Lichtheimia corymbifera 272, and Trichophyton interdigitale 445. All strains, except ATCC one tested, are clinical isolates obtained from the Department of Clinical Microbiology, University Hospital and Faculty of Medicine, Charles University, Prague, Czech Republic. Before testing, each strain was subcultured on Sabouraud dextrose agar (SDA; Difco/Becton Dickinson, Detroit, MI, USA) and maintained on the same medium at $4{ }^{\circ} \mathrm{C}$. Fungal inocula were prepared by suspending yeasts, conidia, or sporangiospores in sterile $0.85 \%$ saline. The cell density was adjusted using a Bürker's chamber to yield a stock 
suspension of $1.0 \pm 0.2 \times 10^{5}$ colony forming units $(\mathrm{CFU}) / \mathrm{mL}$ and $1.0 \pm 0.2 \times 10^{6} \mathrm{CFU} / \mathrm{mL}$ for yeasts and molds, respectively. The final inoculum was made by 1:20 dilution of the stock suspension with the test medium. The compounds were dissolved in DMSO, and the antifungal activity was determined in RPMI 1640 media (KlinLab, Prague, Czech Republic) buffered to pH 7.0 with $0.165 \mathrm{M}$ 3-morpholinopropane-1-sulfonic acid (Sigma-Aldrich, St. Louis, MO, USA). Controls consisted of medium and DMSO alone. The final concentration of DMSO in the test medium did not exceed $1 \%(v / v)$ of the total solution. The concentrations of the studied substances ranged from 500 to $0.488 \mu \mathrm{mol} / \mathrm{L}$. The minimum inhibitory concentration (MIC), was defined as $80 \%$ or greater (for yeasts and yeast-like organisms- $\mathrm{IC}_{80}$ ), resp. $50 \%$ or greater (for molds- $-\mathrm{IC}_{50}$ ) reduction of growth in comparison with the control. The values of MICs were determined after 24 and $48 \mathrm{~h}$ of static incubation at $35^{\circ} \mathrm{C}$. In the case of T. interdigitale, the MICs were recorded after 72 and $120 \mathrm{~h}$ due to its slow growth rate. Fluconazole and voriconazole were used as reference antifungal drugs.

\subsubsection{Evaluation of In Vitro Antibacterial Activity}

The antibacterial activity of all compounds was evaluated by the microdilution broth method [46]. The organisms examined included strains from Czech Collection of Microorganisms (Brno, Czech Republic): Staphylococcus aureus CCM 4516/08 (SA), Escherichia coli CCM 4517 (EC), Pseudomonas aeruginosa CCM 1961 (PA). These strains are recommended as standards for testing of antibacterial activities. Other strains were clinical isolates (Department of Clinical Microbiology, University Hospital and Faculty of Medicine in Hradec Králové, Charles University in Prague, Czech Republic): Staphylococcus aureus H 5996/08 (methicillin resistant, MRSA), Staphylococcus epidermidis H 6966/08 (SE), Enterococcus sp. J 14365/08 (EF), Klebsiella pneumoniae D11750/08 (KP), Klebsiella pneumoniae J 14368/08 (ESBL positive, KP-E). All strains were subcultured on Mueller-Hinton agar (MHA) (Difco/Becton Dickinson, Detroit, MI) at $35{ }^{\circ} \mathrm{C}$ and maintained on the same medium at $4{ }^{\circ} \mathrm{C}$. Prior to testing, each strain was passaged onto MHA. Bacterial inocula were prepared by suspending in sterile $0.85 \%$ saline. The cell density of the inoculum was adjusted using densitometer to yield suspension of density equivalent $0.5 \mathrm{McF}$ arland scale which is equal to a number of $1.5 \times 10^{8} \mathrm{CFU} / \mathrm{mL}$.

The compounds were dissolved in DMSO, and the antibacterial activity was determined in Mueller-Hinton liquid broth (Difco/Becton Dickinson, Detroit, MI, USA), buffered to pH 7.0. Controls consisted of medium and DMSO alone. The final concentration of DMSO in the test medium did not exceed $1 \%(v / v)$ of the total solution composition. The minimum inhibitory concentration (MIC), defined as $95 \%$ inhibition of bacterial growth as compared to control, was determined after 24 and $48 \mathrm{~h}$ of static incubation at $35^{\circ} \mathrm{C}$. Neomycin, bacitracin and penicillin have been used as reference antibacterial drugs.

3.2.3. Evaluation of In Vitro Antimycobacterial Activity on Mycobacterium tuberculosis H37RV CNCTC My 331/88, M. kansasii Hauduroy CNCTC My 235/80 and M. avium ssp. avium Chester CNCTC My $80 / 72$

Microdilution panel method was used. Tested strains Mycobacterium tuberculosis H37RV CNCTC My 331/88, M. kansasii Hauduroy CNCTC My 235/80 and M. avium ssp. avium Chester CNCTC My 80/72 were obtained from Czech National Collection of Type Cultures (CNCTC), National Institute of Public Health, Prague, Czech Republic. Middlebrook 7H9 broth (Sigma-Aldrich, Steinheim, Germany) enriched with $0.4 \%$ of glycerol (Sigma-Aldrich) and $10 \%$ of OADC supplement (oleic acid, albumin, dextrose, catalase; Himedia, Mumbai, India) of declared pH 6.6 was used for cultivation. Tested compounds were dissolved and diluted in DMSO and mixed with broth $(25 \mu \mathrm{L}$ of DMSO solution in $4.475 \mathrm{~mL}$ of broth) and placed $(100 \mu \mathrm{L})$ into microtitration plate wells. Mycobacterial inocula were suspended in isotonic saline solution and the density was adjusted to $0.5-1.0$ McFarland. These suspensions were diluted by $10^{-1}$ and used to inoculate the testing wells, adding $100 \mu \mathrm{L}$ of suspension to $100 \mu \mathrm{L}$ of the DMSO/broth solution of tested compound. Final concentrations of tested compounds in wells were 100, 50, 25, 12.5, 6.25, 3.13 and $1.56 \mu \mathrm{g} / \mathrm{mL}$. Isoniazid was used as positive 
control (inhibition of growth). Negative control consisted of broth plus DMSO. $30 \mu \mathrm{L}$ of Alamar Blue working solution (1:1 mixture of $0.01 \%$ resazurin sodium salt (aq. sol.) and $10 \%$ aqueous solution of Tween 80) was added usually after 5 days of incubation. Results were then determined after $24 \mathrm{~h}$ of incubation and interpreted according to Franzblau [47]. The MIC ( $\mu \mathrm{g} / \mathrm{mL})$ was determined as the lowest concentration which prevented the blue to pink colour change.

\subsubsection{Evaluation of In Vitro Antimycobacterial Activity on Mycobacterium smegmatis}

An antimycobacterial assay was performed with fast growing Mycobacterium smegmatis CCM 4622 (ATCC 607) from Czech Collection of Microorganisms (Brno, Czech Republic). The technique used for activity determination was microdilution broth panel method using 96-well microtitration plates. Culturing medium was Middlebrook 7H9 (MB) broth (Sigma-Aldrich) enriched with $0.4 \%$ of glycerol (Sigma-Aldrich) and 10\% of Middlebrook OADC growth supplement (Himedia, Mumbai, India). Tested compounds were dissolved in DMSO (Sigma-Aldrich) then MB broth was added to obtain concentration $2000 \mu \mathrm{g} / \mathrm{mL}$. Standards used for activity determination were isoniazid (INH), rifampicin (RIF) and ciprofloxacin (CPX) (Sigma-Aldrich). Final concentrations were reached by twofold dilution and addition of mycobacterial suspension and were set as 500, 250, 125, 62.5, 31.25, 15.625, 7.81 and $3.91 \mu \mathrm{g} / \mathrm{mL}$ except to standards ciprofloxacin and rifampicin where the final concentrations were $12.5,6.25,3.125,1.56,0.78,0.39,0.195$ and $0.098 \mu \mathrm{g} / \mathrm{mL}$. Drug-free controls consisted of broth and DMSO and were used as a growth control. The final concentration of DMSO did not exceeded $2.5 \%$ $(v / v)$ to not affect the growth of M. smegmatis. Plates were sealed with polyester adhesive film and incubated in the dark at $37^{\circ} \mathrm{C}$ without agitation. The addition of $0.01 \%$ solution of resazurin sodium salt followed after $48 \mathrm{~h}$. This stain was prepared by dissolving resazurin sodium salt (Sigma-Aldrich) in deionised water to get $0.02 \%$ solution. Then $10 \%$ aqueous solution of Tween 80 (Sigma-Aldrich) was prepared. Both liquids were mixed up making use of the same volumes and filtered through syringe membrane filter. Microtitration plates were then incubated for further $4 \mathrm{~h}$. Antimycobacterial activity was expressed as MIC and the value was determined on the basis of stain color change (blue color-active compound; pink color-non active compound). MIC values for standards were within the ranges 7.81-15.625 $\mu \mathrm{g} / \mathrm{mL}$ for INH, $0.78-1.56 \mu \mathrm{g} / \mathrm{mL}$ for RIF and 0.098-0.195 $\mu \mathrm{g} / \mathrm{mL}$ for CPX. All experiments were conducted in duplicate.

\subsection{Calculation of Lipophilicity}

Theoretical lipophilicities $\log \mathrm{P}$ and corrigated values $\mathrm{C} \log \mathrm{P}$ of all compounds have been calculated in ChemDraw Professional 15.0, part of ChemOffice (Perkin Elmer, Waltham, MA, USA).

\section{Conclusions}

Chalcones have a very simple chemistry, which enables multiplicity of substitutions with easy synthesis and different pharmacological potential in dependence on particular structural modification [13]. Within this work, twenty halogenated pyrazine-based compounds have been prepared and characterized. They are all novel compounds with exception of three compounds. They were tested on antifungal, antibacterial, as well as on antimycobacterial effects. 4-Chlorinated series, prepared earlier, have been included in the assays. The results of biological screenings have been compared with our previously published compounds. Importance of an EWG substitution has been confirmed and some compounds displayed comparable or even better inhibitory activity than standard antifungals, antibiotics and antimycobacterial agents.

As far as the structure-activity relationships are concerned, halogen substitution in the ring B of pyrazine-based chalcones proved to have positive influence on antifungal effect against Candida spp. and Trichophyton interdigitale in comparison with our previously prepared series of pyrazine-based chalcones [4,5,31]. Generally, chlorinated and brominated series inhibited growth of fungi more significantly than fluorinated derivatives. 
Halogen substitution in the ring B had positive impact on inhibition of Staphylococcus spp., whereas it did not influence inhibition of other bacteria from the panel. As for specific substitution, 2-chlorinated derivative inhibited markedly growth of $S$. epidermidis. Concerning mycobacteria, halogenated series inhibited significantly growth of M. krusei, M. tuberculosis and M. smegmatis, but they did not strongly influence the growth of M. avium. The inhibiting activity of halogenated series was comparable with that of nitro series prepared previously [4,5]. Derivatives substituted by halogen in position 2 of the ring $B$ and concurrently substituted by tert-butyl in the position 5 of the pyrazine ring showed the highest inhibition of $M$. tuberculosis. Importance of tert-butyl substitution has been observed previously in other series [5]. Presence of isopropyl in position 5 of the pyrazine ring seems to be important in chlorinated or brominated series in the test against $M$. krusei.

Acknowledgments: The study was supported by Ministry of Education, Youth and Sports of the Czech Republic, projects SVV 260291 and SVV 260289.

Author Contributions: Marta Kucerova-Chlupacova, Veronika Vyskovska-Tyllova and Lenka Richterova-Finkova carried out syntheses, interpreted yields, elemental analysis, IR spectra, and antimycobacterial data; Jiri Kunes recorded and interpreted NMR data; Vladimir Buchta designed antifungal and antibacterial assays and interpreted their results; Marcela Vejsova performed antifungal and antibacterial assays; Pavla Paterova, Lucia Semelkova and Ondrej Jandourek carried out the antimycobacterial assays, Veronika Opletalova proposed the subject, designed the study and together with Marta Kucerova-Chlupacova wrote the paper. All the authors read and approved the final manuscript.

Conflicts of Interest: The authors declare no conflict of interest.

\section{References}

1. Ni, L.M.; Meng, C.Q.; Sikorski, J.A. Recent advances in therapeutic chalcones. Expert Opin. Ther. Pat. 2004, 14, 1669-1691. [CrossRef]

2. Batovska, D.I.; Todorova, I.T. Trends in utilization of the pharmacological potential of chalcones. Curr. Clin. Pharmacol. 2010, 5, 1-29. [CrossRef] [PubMed]

3. Singh, P.; Anand, A.; Kumar, V. Recent developments in biological activities of chalcones: A mini review. Eur. J. Med. Chem. 2014, 85, 758-777. [CrossRef] [PubMed]

4. Opletalova, V.; Pour, M.; Kunes, J.; Buchta, V.; Silva, L.; Kralova, K.; Chlupacava, M.; Meltrova, D.; Peterka, M.; Poslednikova, M. Synthesis and biological evaluation of (E)-3-(nitrophenyl)-1-(pyrazin-2-yl)prop-2-en-1-ones. Collect. Czechoslov. Chem. Commun. 2006, 71, 44-58. [CrossRef]

5. Kucerova-Chlupacova, M.; Kunes, J.; Buchta, V.; Vejsova, M.; Opletalova, V. Novel pyrazine analogs of chalcones: Synthesis and evaluation of their antifungal and antimycobacterial activity. Molecules 2015, 20, 1104-1117. [CrossRef] [PubMed]

6. Boeck, P.; Leal, P.C.; Yunes, R.A.; Cechinel, V.; Lopez, S.; Sortino, M.; Escalante, A.; Furlan, R.L.E.; Zacchino, S. Antifungal activity and studies on mode of action of novel xanthoxyline-derived chalcones. Arch. Pharm. 2005, 338, 87-95. [CrossRef] [PubMed]

7. Lopez, S.N.; Castelli, M.V.; Zacchino, S.A.; Dominguez, J.N.; Lobo, G.; Charris-Charris, J.; Cortes, J.C.G.; Ribas, J.C.; Devia, C.; Rodriguez, A.M.; et al. In vitro antifungal evaluation and structure-activity relationships of a new series of chalcone derivatives and synthetic analogues, with inhibitory properties against polymers of the fungal cell wall. Bioorg. Med. Chem. 2001, 9, 1999-2013. [CrossRef]

8. Hasan, A.; Rasheed, L.; Malik, A. Synthesis and characterization of variably halogenated chalcones and flavonols and their antifungal activity. Asian J. Chem. 2007, 19, 937-948.

9. Sivakumar, P.M.; Kumar, T.M.; Doble, M. Antifungal activity, mechanism and QSAR studies on chalcones. Chem. Biol. Drug Des. 2009, 74, 68-79. [CrossRef] [PubMed]

10. Opletalova, V. Chalcones and their heterocyclic analogues as potential therapeutic agents of bacterial diseases. Ceská Slov. Farm. 2000, 49, 278-284. [PubMed]

11. Nowakowska, Z. A review of anti-infective and anti-inflammatory chalcones. Eur. J. Med. Chem. 2007, 42, 125-137. [CrossRef] [PubMed]

12. Ritter, M.; Martins, R.M.; Dias, D.; Pereira, C.M.P. Recent advances on the synthesis of chalcones with antimicrobial activities: A brief review. Lett. Org. Chem. 2014, 11, 498-508. [CrossRef] 
13. Mahapatra, D.K.; Bharti, S.K.; Asati, V. Chalcone scaffolds as anti-infective agents: Structural and molecular target perspectives. Eur. J. Med. Chem. 2015, 101, 496-524. [CrossRef] [PubMed]

14. Abdullah, M.I.; Mahmood, A.; Madni, M.; Masood, S.; Kashif, M. Synthesis, characterization, theoretical, anti-bacterial and molecular docking studies of quinoline based chalcones as a DNA gyrase inhibitor. Bioorg. Chem. 2014, 54, 31-37. [CrossRef] [PubMed]

15. Chen, Z.H.; Zheng, C.J.; Sun, L.P.; Piao, H.R. Synthesis of new chalcone derivatives containing a rhodanine-3-acetic acid moiety with potential anti-bacterial activity. Eur. J. Med. Chem. 2010, 45, 5739-5743. [CrossRef] [PubMed]

16. Jin, X.; Zheng, C.J.; Song, M.X.; Wu, Y.; Sun, L.P.; Li, Y.J.; Yu, L.J.; Piao, H.R. Synthesis and antimicrobial evaluation of L-phenylalanine-derived C5-substituted rhodanine and chalcone derivatives containing thiobarbituric acid or 2-thioxo-4-thiazolidinone. Eur. J. Med. Chem. 2012, 56, 203-209. [CrossRef] [PubMed]

17. Liu, X.F.; Zheng, C.J.; Sun, L.P.; Liu, X.K.; Piao, H.R. Synthesis of new chalcone derivatives bearing 2,4-thiazolidinedione and benzoic acid moieties as potential anti-bacterial agents. Eur. J. Med. Chem. 2011, 46, 3469-3473. [CrossRef] [PubMed]

18. Lin, Y.M.; Zhou, Y.S.; Flavin, M.T.; Zhou, L.M.; Nie, W.G.; Chen, F.C. Chalcones and flavonoids as anti-tuberculosis agents. Bioorg. Med. Chem. 2002, 10, 2795-2802. [CrossRef]

19. Medvecky, R.; Durinda, J.; Odlerova, Z.; Polasek, E. Antimykobakteriálna aktivita azachalkónov, ich derivátov a analogických látok I. Farm. Obzor. 1992, 61, 341-350.

20. Fanzani, L.; Porta, F.; Meneghetti, F.; Villa, S.; Gelain, A.; Lucarelli, A.P.; Parisini, E. Mycobacterium tuberculosis low molecular weight phosphatases (MPtpA and MPtpB): From biological insight to inhibitors. Curr. Med. Chem. 2015, 22, 3110-3132. [CrossRef] [PubMed]

21. Anand, N.; Singh, P.; Sharma, A.; Tiwari, S.; Singh, V.; Singh, D.K.; Srivastava, K.K.; Singh, B.N.; Tripathi, R.P. Synthesis and evaluation of small libraries of triazolylmethoxy chalcones, flavanones and 2-aminopyrimidines as inhibitors of mycobacterial FAS-II and PknG. Bioorg. Med. Chem. 2012, 20, 5150-5163. [CrossRef] [PubMed]

22. Lim, S.S.; Kim, H.S.; Lee, D.U. In vitro antimalarial activity of flavonoids and chalcones. Bull. Korean Chem. Soc. 2007, 28, 2495-2497.

23. Hasan, S.A.; Elias, A.N.; Jwaied, A.H.; Khuodaer, A.R.; Hussain, S.A. Synthesis of new fluorinated chalcone derivatives with anti-inflammatory activity. Int. J. Pharm. Pharm. Sci. 2012, 4, 430-434.

24. Rojas, J.; Paya, M.; Dominguez, J.N.; Ferrandiz, M.L. The synthesis and effect of fluorinated chalcone derivatives on nitric oxide production. Bioorg. Med. Chem. Lett. 2002, 12, 1951-1954. [CrossRef]

25. Bukhari, S.N.A.; Jantan, I.; Jasamai, M. Anti-inflammatory trends of 1,3-diphenyl-2-propen-1-one derivatives. Mini Rev. Med. Chem. 2013, 13, 87-94. [CrossRef] [PubMed]

26. Mahapatra, D.M.; Bharti, S.K.; Asati, V. Anti-cancer chalcones: Structural and molecular target perspectives. Eur. J. Med. Chem. 2015, 98, 69-114. [CrossRef] [PubMed]

27. Bois, F.; Beney, C.; Boumendjel, A.; Mariotte, A.M.; Conseil, G.; Di Pietro, A. Halogenated chalcones with high-affinity binding to p-glycoprotein: Potential modulators of multidrug resistance. J. Med. Chem. 1998, 41, 4161-4164. [CrossRef] [PubMed]

28. Kabir, A.M.A.; Shimizu, K.; Aiba, Y.; Igarashi, M.; Takagi, A.; Koga, Y. The effect of sofalcone on indomethacin-induced gastric ulcers in a Helicobacter pylori-infected gnotobiotic murine model. Aliment. Pharmacol. Ther. 2000, 14, 223-229. [CrossRef] [PubMed]

29. Opletalova, V.; Patel, A.; Boulton, M.; Dundrova, A.; Lacinova, E.; Prevorova, M.; Appeltauerova, M.; Coufalova, M. 5-Alkyl-2-pyrazinecarboxamides, 5-alkyl-2-pyrazinecarbonitriles and 5-alkyl-2-acetylpyrazines as synthetic intermediates for antiinflammatory agents. Collect. Czechoslov. Chem. Commun. 1996, 61, 1093-1101. [CrossRef]

30. Kucerova-Chlupacova, M.; Opletalova, V.; Jampilek, J.; Dolezel, J.; Dohnal, J.; Pour, M.; Kunes, J.; Vorisek, V. New hydrophobicity constants of substituents in pyrazine rings derived from RP-HPLC study. Collect. Czechoslov. Chem. Commun. 2008, 73, 1-18. [CrossRef]

31. Opletalova, V.; Hartl, J.; Patel, A.; Palat, K.; Buchta, V. Ring substituted 3-phenyl-1-(2-pyrazinyl)-2-propen-1-ones as potential photosynthesis-inhibiting, antifungal and antimycobacterial agents. Farmaco 2002, 57, 135-144. [PubMed] 
32. Chlupacova, M.; Opletalova, V.; Kunes, J.; Silva, L.; Buchta, V.; Duskova, L.; Kralova, K. Synthesis and biological evaluation of some ring-substituted (E)-3-aryl-1-pyrazin-2-ylprop-2-en-1-ones. Folia Pharm. Univ. Carol. 2005, 31-43.

33. Opletalová, V.; Chlupáčová, M.; Buchta, V.; Silva, L. Antifungal properties of chalcones and their heterocyclic analogues. In International Symposium on Natural Drugs; Borelli, F., Capasso, F., Milic, N., Russo, A., Eds.; Universita degli Studi di Napoli Federico II, Naples-Indena: Naples, Italy, 2003; pp. 259-261.

34. Chlupáčová, M. Chalcone and Their Analogues as Potential Drugs. Ph.D. Thesis, Charles University in Prague, Hradec Králové, Czech Republic, 2006.

35. Peterka, M. Chalcones and Their Analogues as Potential Drug I. Diploma Thesis, Charles University in Prague, Hradec Králové, Czech Republic, 2000.

36. Gupta, A.K.; Kohli, Y.; Batra, R. In vitro activities of posaconazole, ravuconazole, terbinafine, itraconazole and fluconazole against dermatophyte, yeast and non-dermatophyte species. Med. Mycol. 2005, 43, 179-185. [CrossRef] [PubMed]

37. Gabor, M.; Sallai, J.; Szell, T.; Sipos, G. Relation of antibacterial activity and chemical structure of chalcone derivatives. Acta Microbiol. Acad. Sci. Hung. 1967, 14, 45-64. [PubMed]

38. Nielsen, S.F.; Boesen, T.; Larsen, M.; Schonning, K.; Kromann, H. Antibacterial chalcones-bioisosteric replacement of the $4^{\prime}$-hydroxy group. Bioorg. Med. Chem. 2004, 12, 3047-3054. [CrossRef] [PubMed]

39. Rizvi, S.U.F.; Siddiqui, H.L.; Parvez, M.; Ahmad, M.; Siddiqui, W.A.; Yasinzai, M.M. Antimicrobial and antileishmanial studies of novel (2E)-3-(2-chloro-6-methyl/methoxyquinolin-3-yl)-1-(aryl)prop-2-en-1-ones. Chem. Pharm. Bull. 2010, 58, 301-306. [CrossRef] [PubMed]

40. Rane, R.A.; Telvekar, V.N. Synthesis and evaluation of novel chloropyrrole molecules designed by molecular hybridization of common pharmacophores as potential antimicrobial agents. Bioorg. Med. Chem. Lett. 2010, 20, 5681-5685. [CrossRef] [PubMed]

41. Liaras, K.; Geronikaki, A.; Glamoclija, J.; Ciric, A.; Sokovic, M. Thiazole-based chalcones as potent antimicrobial agents. Synthesis and biological evaluation. Bioorg. Med. Chem. 2011, 19, 3135-3140. [CrossRef] [PubMed]

42. Metzner, J.; Zamocka, J.; Heger, J. Anti-microbial studies on diazachalkones and 2-pyrazoline derivatives. Pharmazie 1981, 36, 157-157. [PubMed]

43. Zámocká, J.; Dvořáčková, D.; Heger, J. Darstellung und Strukturbestimmung einiger Diazachalkone. Z. Chem. 1980, 20, 29-30. [CrossRef]

44. Clinical and Laboratory Standards Institute. Reference Method for Broth Dilution Antifungal Susceptibility Testing of Yeasts: Approved Standard, CLSI Document M27-A3, 3rd ed.; Clinical and Laboratory Standards Institute: Wayne, PA, USA, 2008.

45. Clinical and Laboratory Standards Institute. Reference Method for Broth Dilution Antifungal Susceptibility Testing of Filamentous Fungi: Approved Standard, CLSI Document M38-A2, 2nd ed.; Clinical and Laboratory Standards Institute: Wayne, PA, USA, 2008.

46. Jones, R.N.; Barry, A.L. Optimal dilution susceptibility testing conditions, recommendations for MIC interpretation, and quality-control guidelines for the ampicillin-sulbactam combination. J. Clin. Microbiol. 1987, 25, 1920-1925. [PubMed]

47. Franzblau, S.G.; Witzig, R.S.; McLaughlin, J.C.; Torres, P.; Madico, G.; Hernandez, A.; Degnan, M.T.; Cook, M.B.; Quenzer, V.K.; Ferguson, R.M.; et al. Rapid, low-technology MIC determination with clinical mycobacterium tuberculosis isolates by using the microplate Alamar Blue assay. J. Clin. Microbiol. 1998, 36, 362-366. [PubMed]

Sample Availability: Samples of the compounds are available from the authors. 